\title{
A 3D sunspot model derived from an inversion of spectropolarimetric observations and its implications for the penumbral heating ${ }^{\star}$
}

\author{
C. Beck $^{1,2}$ \\ 1 Instituto de Astrofísica de Canarias (CSIC),Via Lactea, 38205 La Laguna, Tenerife, Spain \\ e-mail: cbeck@iac.es \\ 2 Kiepenheuer-Institut für Sonnenphysik, Schöneckstr. 6, 79104 Freiburg, Germany
}

Received 3 August 2007 / Accepted 30 November 2007

ABSTRACT

\begin{abstract}
Aims. I deduced a 3D sunspot model that is in full agreement with spectropolarimetric observations, in order to address the question of a possible penumbral heating process by the repetitive rise of hot flow channels.

Methods. I performed inversions of spectropolarimetric data taken simultaneously in infrared $(1.5 \mu \mathrm{m})$ and visible $(630 \mathrm{~nm})$ spectral lines. I used two independent magnetic components inside each pixel to reproduce the irregular Stokes profiles in the penumbra and studied the averaged and individual properties of the two components. By integrating the field inclination to the surface, I developed a 3D model of the spot from inversion results without intrinsic height information.

Results. I find that the Evershed flow is harbored by the weaker of the two field components. This component forms flow channels that show upstreams in the inner and mid penumbra, continue almost horizontally as slightly elevated loops throughout the penumbra, and finally bend down in the outer penumbra. I find several examples where two or more flow channels are found along a radial cut from the umbra to the outer boundary of the spot.

Conclusions. I find that a model of horizontal flow channels in a static background field is in good agreement with the observed spectra. The properties of the flow channels correspond very well to the moving tube simulations of Schlichenmaier et al. (1998, A\&A, 337, 897). From the temporal evolution in intensity images and the properties of the flow channels in the inversion, I conclude that interchange convection of rising hot flux tubes in a thick penumbra still seems a possible mechanism for maintaining the penumbral energy balance.
\end{abstract}

Key words. Sun: magnetic fields - Sun: sunspots

\section{Introduction}

Sunspots were the first indicators of solar magnetic activity already detected centuries ago in the western world (Galilei et al. 1613; Galilei 1632) and millennia ago in Asia (cf. Wittmann \& $\mathrm{Xu}$ 1987). Their magnetic nature was only proven in the last century by Hale (1908). Using spectroscopic observations, Evershed (1909) showed that sunspots exhibit a particular flow field in the penumbra, the brighter halo surrounding the dark umbra: as soon as a sunspot was observed not directly on disc center, spectral lines in the half of the spot facing disc center were seen to be blue-shifted, whereas they were red-shifted in the half facing the limb. This "Evershed flow" was explained as the signature of a radial outflow, which had to be close to parallel to the solar surface.

Despite its rather well defined spectroscopic properties, the Evershed flow still eludes a generally accepted explanation. The reason is the fine structure of the sunspot's penumbra that became visible when both the spatial resolution and the information content of observations were improved. Spectroscopic observations with high spatial resolution show that the Evershed flow is organized in many small-scale radially oriented filaments, which carry the bulk of the flow, whereas in the space

\footnotetext{
* Movies and Appendices A-C are only available in electronic form at http://www . aanda.org
}

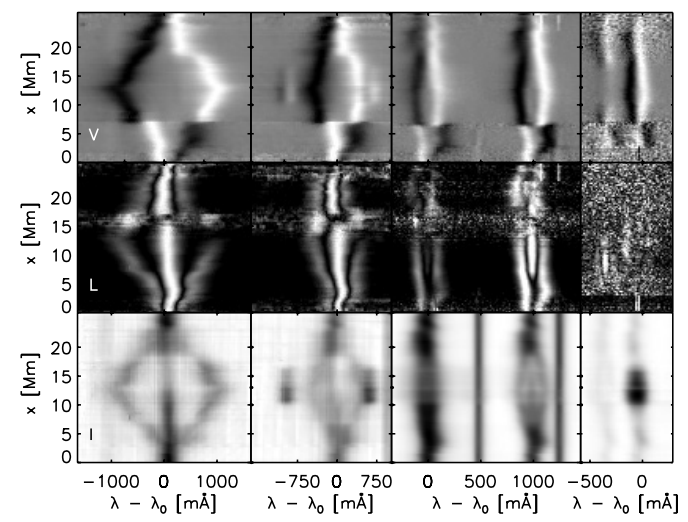

Fig. 1. Stokes profiles along the symmetry line connecting spot center and disc center. The limb side is down, the center side up. Bottom row: intensity, I. Middle row: total linear polarization, L. Top row: circular polarization, $V$. Left to right: IR spectral lines at $1564.8 \mathrm{~nm}, 1565.2 \mathrm{~nm}$, visible lines at $630.15 \mathrm{~nm}, 630.25 \mathrm{~nm}$, and Ti I at $630.37 \mathrm{~nm}$.

in between the flow velocity is strongly reduced (Tritschler et al. 2004; Langhans et al. 2005; Rimmele \& Marino 2006). Different, and in some cases contradictory results were found for the correlation between these flow filaments and intensity filaments (cf. the discussion in Langhans et al. 2005). 

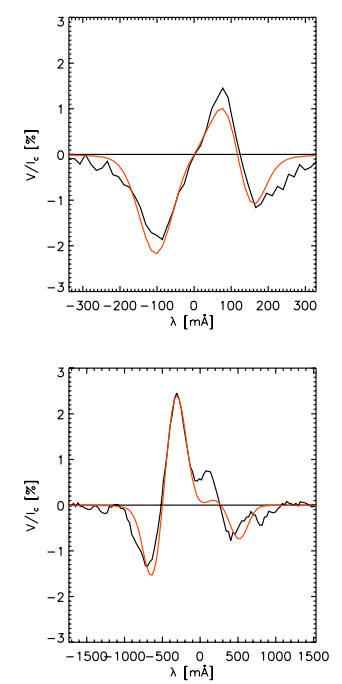
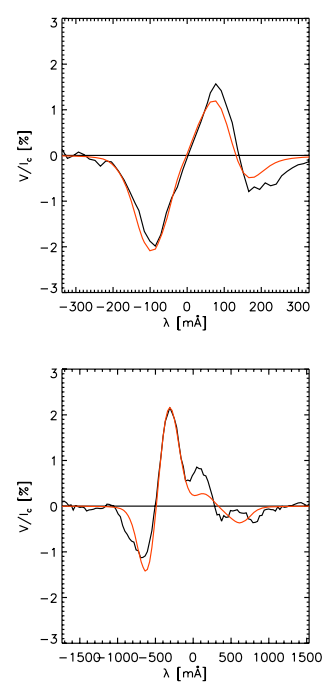
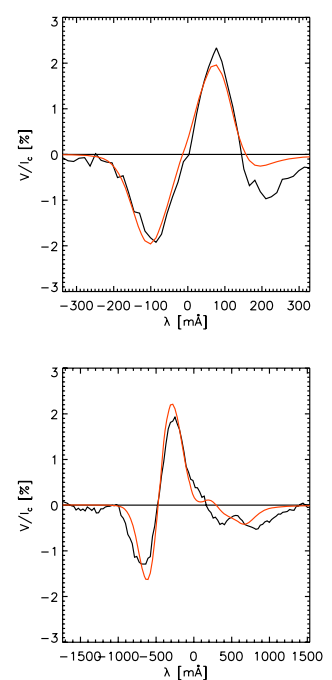
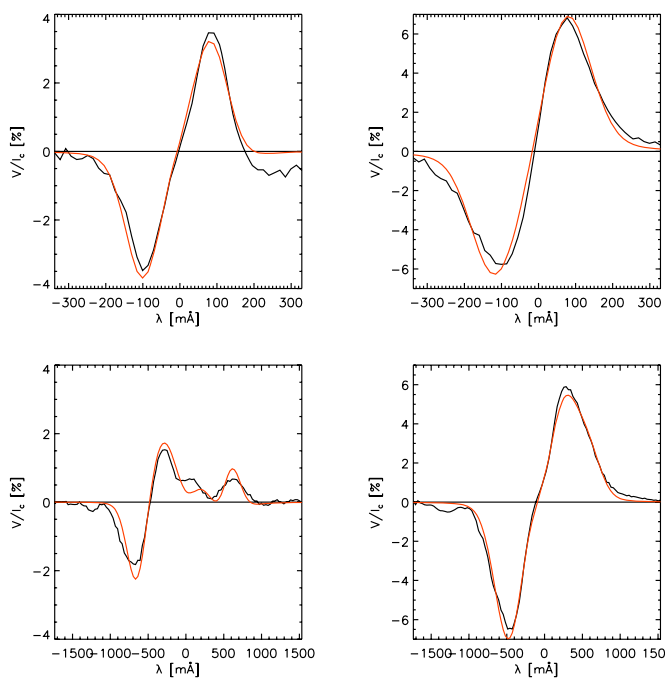

Fig. 2. Examples of Stokes $V$ profiles in the neutral line. Top row: Stokes $V$ of the Fe I line at $630.15 \mathrm{~nm}$. Bottom row: the co-spatial profiles of Fe I at $1564.8 \mathrm{~nm}$. The red line gives the corresponding best-fit profile of the inversion. The last column shows a profile in the center side penumbra.

Using spectropolarimetric observations of sunspots, the information content of the observations was extended to encompass both the magnetic field topology and the flow fields at the same time. The problem of the penumbral fine structure, however, was not resolved by this, but became still more complex. The Evershed flow could be shown to be close to horizontal, but the average magnetic field turned out not to be (e.g. Bellot Rubio et al. 2003). A possible explanation for the mismatch between flow and field direction in an ionized plasma is the assumption that, at the spatial resolution of around 1", achieved in current spectropolarimetric observations, two different magnetic field components are seen inside the same pixel. These two components have a different topology and flow field each.

One way to visualize this is to make a cut along the symmetry line of a sunspot, the line that passes from disc center across the spot center towards the closest solar limb position (Collados 2002; Bellot Rubio et al. 2004). Figure 1 shows profiles of intensity, total linear polarization, $L=\sqrt{Q^{2}+U^{2}}$, and circular polarization (Stokes $V$ ) along the symmetry line for one of the observations analyzed in the present study. Both circular and linear polarizations show a neutral line on the limb side and center side, respectively. At the location of these neutral lines, the profile shape changes abruptly. On the limb side across the neutral line of Stokes $V$, stronger fields with smaller line shifts turn into weaker fields with larger line shifts. On the center side across the neutral line of $L$, the same change is seen from strong fields with slower flows to weaker fields with stronger flows.

More direct evidence of these multiple magnetic field components is given by the Stokes $V$ profiles close to or within the neutral line of $V$ (Fig. 2) on the limb side. The neutral line indicates a local minimum of the Stokes $V$ signal amplitude, because most of the field lines are perpendicular to the line of sight (LOS). In magnetograms, the retrieved signal passes through zero in the neutral line, because the polarity of the magnetic field changes, e.g. from positive to negative, but the $V$ signal does not disappear completely in spectra due to the multicomponent penumbral structure (cf. Schlichenmaier \& Collados 2002). These profiles in the neutral line show not only two $\sigma$-components in the circular polarization signal, but several local extrema (both minima and maxima). To generate such complex patterns, the magnetic field has to be complex as well. Appendix A shows how such complex profiles can be created by the addition of two regular Stokes $V$ profiles with opposite polarities. This simple addition of profiles, however, does not tell whether the two magnetic field components leading to the $V$ profiles are actually located in the same pixel. If the spatial resolution of the polarimetric observations is insufficient for separating the flow filaments seen in high-resolution observations from their surroundings, field components from two different spatial locations would be added up.

There is, however, an indicator that suggests that the field components are not spatially separated in the horizontal dimensions, but vertically interlaced, the net circular polarization (NCP). A non-zero value of NCP - as observed in the penumbra of sunspots (e.g. Sánchez Almeida \& Lites 1992; Solanki \& Montavon 1993; Martínez Pillet 2000; Müller et al. 2002, 2006) - indicates discontinuities, or at least strong gradients of field properties, along the line of sight inside the formation height of spectral lines.

Most of the observed properties of profiles in the penumbra of sunspots can be explained with the uncombed penumbra model suggested by Solanki \& Montavon (1993). In this model, a more vertical field component winds around horizontal flow channels, which carry the Evershed flow. The success of this model is related to its being able to explain the most prominent peculiarities of sunspots: a horizontal flow field in a nonhorizontal field topology, the appearances of neutral lines in total linear polarization, $L$, and Stokes $V$, and the NCP. However, the model itself gives no explanation, why its specific topology should exist in the penumbra. The simulations of Schlichenmaier et al. (1998) show that such a configuration of embedded flow channels results from the temporal evolution of magnetic flux initially located at the boundary layer between the sunspot and the field-free surroundings. Thomas \& Weiss (2004) suggest that turbulent convection outside the spot pulls down field lines and thus produces the filamentary structure of the sunspot. In both cases, the penumbra consists of similar embedded flow channels, but created by different mechanisms. Spruit \& Scharmer (2006) and Scharmer \& Spruit (2006) have recently suggested a completely different model where the intensity filaments are related to the existence of field-free gaps below the visible surface layer.

In this paper, I want to show that a rather simple uncombed model using two depth-independent magnetic components is 

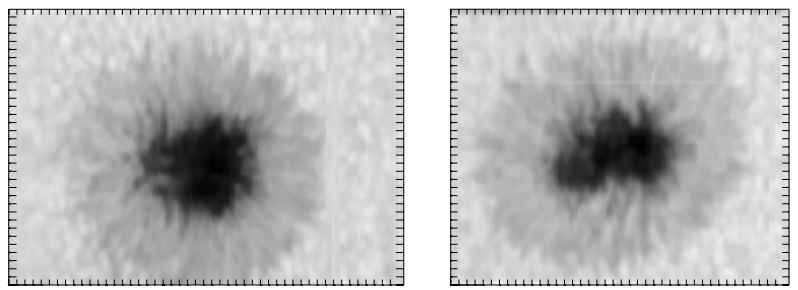

Fig. 3. Continuum intensity maps of NOAA 10425 in the near infrared on 2003 August 7 (left) and August 9 (right). The distance between tick marks is 1 arcsec.

sufficient for reproducing simultaneous observations in infrared and visible spectral lines with their different responses to magnetic fields. The inversion setup is described after the observations and data reduction (Sect. 2). I study average field properties and their radial variation in Sect. 4 and then deduce a geometrical model of the sunspot by integrating the surface inclination of the fields (Sect. 5). The physical properties of the model, such as field strength, velocity, or field orientation, correspond to the best-fit model atmospheres needed to reproduce the observed spectra. I briefly investigate the temporal evolution in Sect. 6, with emphasis on which parts change with time and which do not. In the discussion (Sect. 7), I address the question of penumbral heat transport in the context of the results of the previous analysis. Appendix A shows how complex profiles can be created with simple assumptions. Appendix B shows an example of the integration along a single cut through the penumbra and the full 3D model from different viewing angles. Appendix C describes the analysis of a time series of speckle-reconstructed G-band images used to derive the characteristic time scale of penumbral intensity variations.

I used a simple inversion setup with field properties constant with optical depth, corresponding to a horizontal separation of the field components. A more sophisticated setup, taking the variations along the line of sight into account, is planned for discussion in a forthcoming paper.

\section{Observations and data reduction}

For this work, two observations of the sunspot NOAA 10425 were analyzed. They were taken on 2003 August 7 and 9 with the two vector spectropolarimeters of the German Vacuum Tower Telescope (VTT) on Tenerife: the Polarimetric Littrow Spectrograph (POLIS; Beck et al. 2005b) and the Tenerife Infrared Polarimeter (TIP; Martínez Pillet et al. 1999). The two instruments were used simultaneously with an achromatic 5050-beamsplitter. During the observations, POLIS was remotecontrolled by TIP to ensure strictly simultaneous exposures. The image motion was reduced with the Correlation Tracker System (Schmidt \& Kentischer 1995; Ballesteros et al. 1996). The Stokes vector in the visible and infrared (IR) spectral lines listed in Table 1 was retrieved. An integration time of around $3.5 \mathrm{~s}$ was used for each slit position. The slit width was 0.'36 for TIP and 0.'5 for POLIS. The scanning step width was 0.36. Spatial sampling along the slit was 0.37 for TIP and 0.145 for POLIS. The POLIS data were interpolated later to have the same spatial sampling along the slit as TIP. Figure 3 displays maps of the continuum intensity in the infrared. The 2D intensity maps were constructed from the intensity along the slit for each scan step, the scanning direction is left to right. The heliocentric angle of the spot on August 7 and 9 was $7^{\circ}$ and $30^{\circ}$, respectively. The spatial resolution was estimated from the spatial
Table 1. Spectral lines observed with POLIS (top) and TIP (bottom).

\begin{tabular}{lcccc}
\hline \hline $\begin{array}{l}\text { Element } \\
\text { ion. state }\end{array}$ & $\begin{array}{c}\lambda \\
{[\mathrm{nm}]}\end{array}$ & $\begin{array}{c}\text { Transition } \\
{ }^{2 S+1} L_{J}\end{array}$ & $\log (g f)$ & $\begin{array}{c}\text { Landé factor } \\
g_{\text {eff }}\end{array}$ \\
\hline \multicolumn{5}{c}{ POLIS } \\
$\mathrm{Fe} \mathrm{I}$ & $630.15012^{a}$ & ${ }^{5} \mathrm{P}_{2}-{ }^{5} \mathrm{D}_{2}$ & $-0.75^{d}$ & 1.67 \\
$\mathrm{Fe} \mathrm{I}$ & $630.24936^{a}$ & ${ }^{5} \mathrm{P}_{1}-{ }^{5} \mathrm{D}_{0}$ & $-1.236^{b}$ & 2.50 \\
$\mathrm{Fe} \mathrm{I}^{b}$ & 630.34600 & ${ }^{5} \mathrm{G}_{6}-{ }^{5} \mathrm{G}_{5}$ & -2.55 & 1.50 \\
$\mathrm{Ti} \mathrm{I}^{b}$ & 630.37525 & ${ }^{3} \mathrm{~F}_{3}-{ }^{3} \mathrm{G}_{3}$ & -1.44 & 0.92 \\
& \multicolumn{5}{c}{ TIP } \\
$\mathrm{Fe} \mathrm{I}^{c}$ & 1564.7410 & ${ }^{7} \mathrm{D}_{2}-{ }^{5} \mathrm{P}_{2}$ & -0.95 & 1.25 \\
$\mathrm{Fe} \mathrm{I}^{c}$ & 1564.8515 & ${ }^{7} \mathrm{D}_{1}-{ }^{7} \mathrm{D}_{1}$ & -0.67 & 3 \\
$\mathrm{Fe} \mathrm{I}^{c}$ & 1565.2874 & ${ }^{7} \mathrm{D}_{5}-{ }^{7} \mathrm{D}_{4}$ & $-0.095^{d}$ & 1.45 \\
\hline
\end{tabular}

${ }^{a}$ Nave et al. (1994); ${ }^{b}$ Cabrera Solana et al. (2005); ${ }^{c}$ Bellot Rubio et al. (2000); ${ }^{d}$ Bellot Rubio, priv. comm. All values are given as used in the inversion; they may slightly deviate from the sources cited in $\lambda$ and $\log (g f)$ due to changes in the adopted solar iron abundance and new IR line measurements.

Fourier power spectrum to be around $1^{\prime \prime}$. The data from TIP and POLIS were treated with the flatfielding procedures and polarimetric corrections for instrumental effects (see for example Beck et al. 2005b,a). Residual crosstalk between the different Stokes parameters was estimated to be on the order of $10^{-3} I_{\mathrm{c}}$. The remaining rms noise in the profiles in continuum windows was $4 \times 10^{-4} I_{\mathrm{c}}$ for the IR spectra and $10^{-3} I_{\mathrm{c}}$ for the visible spectra.

The data alignment was done in the same way as described in detail in Beck (2006) or the Appendix of Beck et al. (2007). The wavelength scale was also set up like in the latter, with the blueshift values predicted by the quiet Sun (QS) model of Borrero $\&$ Bellot Rubio (2002) as a reference. The Stokes profiles of each spectral range were normalized to the continuum intensity of the quiet Sun at disk center in a two-step procedure. An average QS profile for each wavelength range was calculated from pixels located outside the sunspot. This profile was normalized to unity with its respective average continuum intensity. The offcenter position was taken into account by multiplying the QS profile and all other profiles with the appropriate limb-darkening coefficient.

\section{Inversion of the Stokes profiles}

The four visible and the three IR lines were inverted together using the SIR code (Stokes Inversion based on Response functions; Ruiz Cobo \& del Toro Iniesta 1992; Ruiz Cobo 1998). To facilitate a good choice for the inversion setup and the initial model components to be used, I created masks of the inversion type. I used the intensity maps in infrared to define the boundaries between umbra and penumbra, and penumbra and surrounding granulation, respectively. Outside the sunspot, I used a threshold in polarization degree to distinguish between field-free pixels and those with a polarization signal large enough to derive the magnetic field. The threshold was set to $0.4 \%$ for the IR spectral lines $(1564.8,1565.2 \mathrm{~nm})$ and $0.75 \%$ for the visible spectral lines $(630.15,630.25 \mathrm{~nm})$. If any one of the lines exceeded its respective threshold, a magnetic field was assumed to be present.

All model atmospheres were prescribed as a function of continuum optical depth, $\tau$, in the range $\log \tau=1$ to -4 . For the initial temperature stratification, I always adopted the HSRA model (Gingerich et al. 1971). Temperature was allowed to be varied with two nodes; i.e., perturbations of the initial model atmosphere with a straight line of arbitrary slope were possible. A 

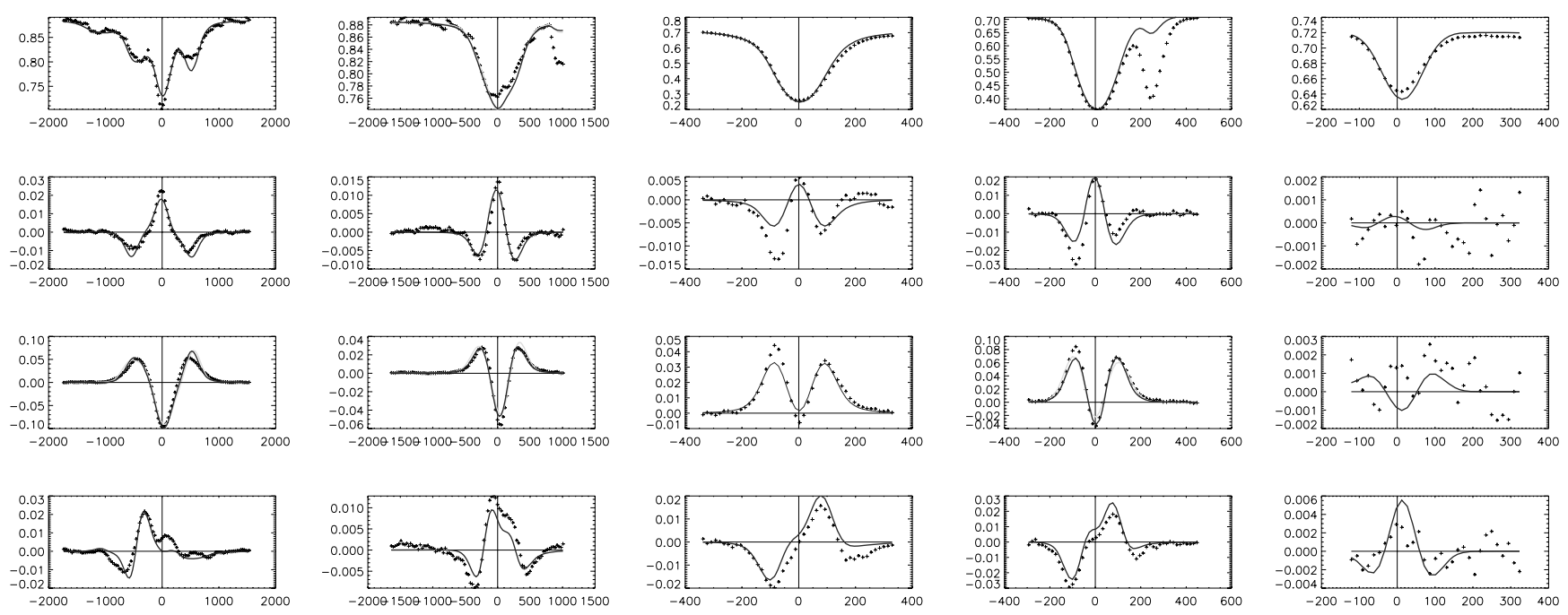

Fig. 4. Comparison of the observed (+) and best-fit profiles (solid line) for one spatial position in the neutral line of Stokes V. Left to right: IR lines at $1564.8 \mathrm{~nm}$ and at $1565.2 \mathrm{~nm}$; visible lines at $630.15 \mathrm{~nm}, 630.25 \mathrm{~nm}$, and $630.37 \mathrm{~nm}$. Top to bottom: Stokes IQUV as a fraction of the continuum intensity. The black vertical lines mark the zero wavelength of the respective wavelength scales. The dispersion on the $x$-axis is in m $\AA$ for all spectra.

contribution of stray light to the observed profiles was also always allowed for; as proxy for the stray light profile the average QS profile was used.

I employed a two-component inversion with one magnetic and one field-free component for pixels outside the sunspot with significant polarization signal. The free parameters of the fieldfree component were the temperature $T$ and LOS velocity $v$. For the magnetic atmosphere component, additional parameters were the field strength $B$, the LOS inclination $\gamma$, and the azimuth of the field in the plane perpendicular to the LOS $\psi$. Except for temperature, all atmospheric parameters were assumed to be constant with optical depth. The spectra, whose polarization degree was below the threshold, were not inverted. I assumed a single magnetic component in the umbra. For all pixels in the penumbra, the inversion setup used two independent magnetic components in each pixel. The macroturbulent velocity was the only parameter that was forced to be equal in both components. All quantities besides $T$ were again constant with depth.

For SIR, the two inversion components are fully equivalent, because the changes applied to their initial model atmospheres are only driven by the need to minimize the deviation between observed and synthetic profiles. In the inversion of the spectra of neighboring pixels, the roles of the two inversion components may thus be exchanged; i.e., component 1 may show a high flow velocity and component 2 is at rest in one case, whereas on the next pixel it is the opposite. The results of the inversion must thus be sorted somehow to provide a smooth spatial variation. I used the inclination to the surface as the criterion for separating the two inversion components into different maps: the more vertical component of each pixel is assumed to be the static background (bg) field of the spot, and the more inclined component to be the flow channels (fc). This selection by a single criterion can lead to ambiguities, when the inclination of the two components is similar. Near the umbra-penumbra boundary, the field inclinations of the two components were nearly identical for both observations of the sunspot (cf. Beck 2006, Fig. 5.14). In this area, I modified the criterion and used the temperature of the components: the hotter component was chosen to be the bg component, the cooler one the fc. This yielded smooth temperature maps, which otherwise showed clear indications that the identification of the components by inclination was wrong.

Figure 4 shows an example of observed and best-fit profiles for one location on the neutral line of Stokes $V$. The weak Fe I line at $630.35 \mathrm{~nm}$ has been left out in the graphs to save some room. The inversion is not able to reproduce asymmetric profiles, and thus fails to retrieve the observed profiles down to the finest details (e.g., Stokes $Q$ and $U$ of the visible lines); however, it still catches the overall shape of the profiles quite well. Especially in Stokes $V$, one and the same atmosphere leads to strongly differing profiles in, e.g., $1564.8 \mathrm{~nm}$ and $630.15 \mathrm{~nm}$. The good agreement of observed and best-fit profiles in both wavelength ranges using constant field properties is related to the only small difference in formation height between the IR and visible lines. Cabrera Solana et al. (2005) studied the properties of several photospheric lines and conclude that the formation height difference between the $1.56 \mu$ lines and those at $630 \mathrm{~nm}$ is less than $100 \mathrm{~km}$. The profiles shown in Fig. 4 have a non-zero $\mathrm{NCP}$, which is not reproduced by the best-fit profiles. Even if the observed sunspot has a non-zero NCP with different behavior in IR and visible spectral lines (Müller et al. 2006), the main information contained in the spectra is at first the average properties of the magnetic field such as field strength. The NCP then yields information on the variation along the LOS, but always around the average value. In Beck (2006), I found that the analysis of the same data set using an uncombed inversion model yielded almost identical average field properties; i.e., field strength or field orientation were not influenced by the choice of the inversion setup with or without reproduction of the NCP. This does not come as a surprise, because the inversion with constant magnetic field properties already reproduces the observed (complex) spectra fairly well (cf. Figs. 2 and 4).

\section{Results}

Prior to further analysis, the inversion results were transformed from the LOS reference frame into the local reference frame (LRF). The LRF is defined such that $z$ corresponds to the surface normal and increases with height, while the $x$-axis points from the center of the spot towards the disc center. The $180^{\circ}$ azimuth 

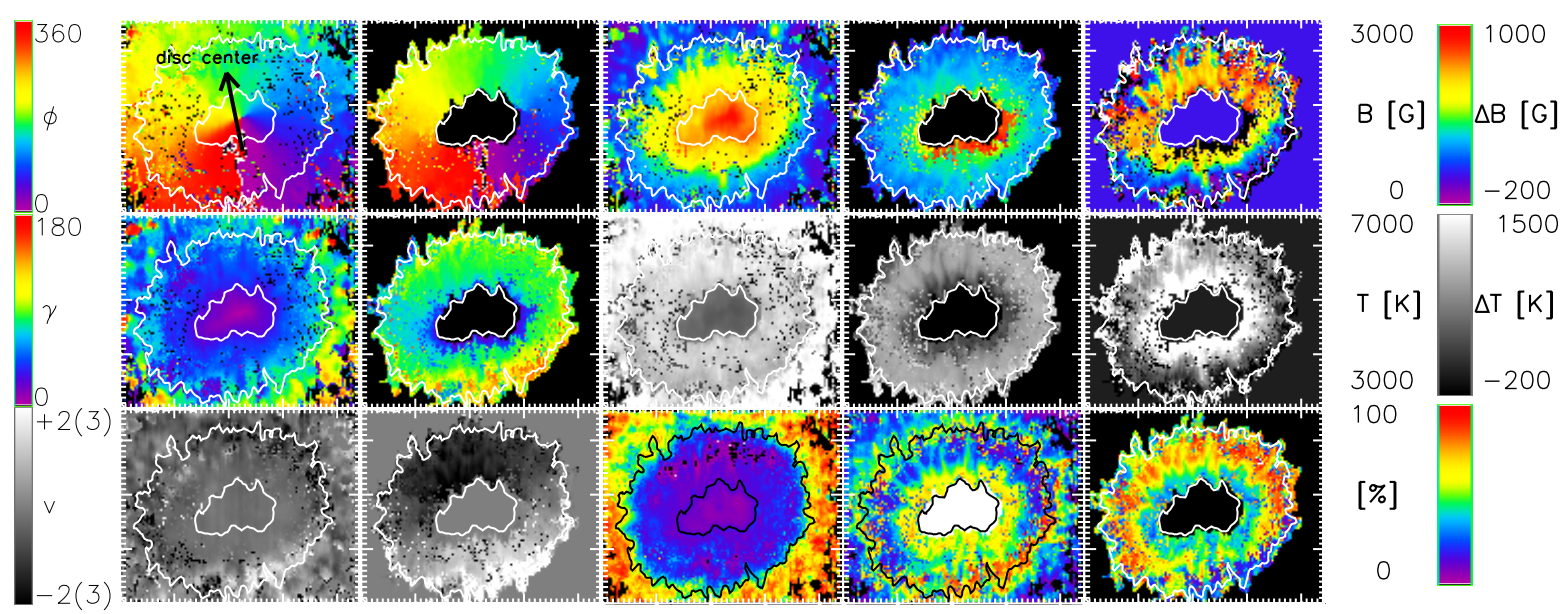

Fig. 5. Top row, left to right: magnetic field azimuth of the background component (bg), field azimuth of the flow-channel component (fc), field strength of $\mathrm{bg}$, field strength of $\mathrm{fc}$, and difference of the field strengths (bg-fc). Middle row, left to right: field inclination of bg and fc, temperature of $\mathrm{bg}$ and $\mathrm{fc}$, difference of temperatures (bg-fc). Bottom row, left to right: LOS velocity of bg and $\mathrm{fc}$, stray light contribution, filling fraction of $\mathrm{bg}$ and fc. Inclination and azimuth are given in the LRF frame; velocities are in $\mathrm{km} \mathrm{s}^{-1}$ with negative velocities pointing towards the observer. The velocity limits in parentheses refer to the fc component. White contour lines denote the inner and outer boundary of the penumbra. The black arrow in the azimuth map points towards disc center.

ambiguity was resolved by assuming a radial field orientation inside the sunspot, and thus always choosing the azimuth solution closer to radial orientation.

\section{1. $2 D$ maps of field parameters}

Figure 5 shows the resulting field azimuth and inclination to the surface normal. Only the results of the observation on August 9 at $30^{\circ}$ are shown here and in the following, as the other observation yielded similar results. The two inversion components have been separated by the criteria described in the previous section. There is an easy way to control whether the selection is reasonable. The component selected as "flow" channels should also exhibit significant flow velocities. And indeed this is the case: the fc component shows LOS velocities up to some $\mathrm{km} \mathrm{s}^{-1}$, whereas the bg component is almost at rest (cf. bottom row of Fig. 5). This gives confidence that the other quantities from the inversion can really be ascribed to "flow channels" and a "background component".

Figure 5 also shows that the bg component is stronger almost throughout the whole penumbra, whereas the field strength becomes comparable at the outer boundary. The patch of increased field strength in the fc component (red color) below and right of the umbra near its boundary to the penumbra is co-spatial to the locations, where the field inclinations of the two components were nearly identical. The strong difference to the field strength further radially outwards could indicate that no clear signatures of two magnetic components were present in the spectra of these locations. The temperature maps (at $\log \tau=0$ ) in the middle row show the bg component to be hotter than the flow channels throughout the whole penumbra. The stray light contribution to the best-fit profiles increases from $10 \%$ in the umbra to $15 \%$ in the penumbra, and strongly increases at the outer spot boundary. Inside the penumbra, flow channels and bg component have a comparable filling fraction of around $50 \%$.

Radially oriented structures ("spines", Lites et al. 1993) can be seen on the center side in the bg components' field strength, in the fc temperature, and in the relative filling fraction of the bg component. The spines start with locally enhanced field strength of the bg component in the umbra, and maintain stronger and less inclined fields throughout the penumbra. The filling fraction of the fc component inside the spines is only $50 \%$, compared to about $70 \%$ outside the spines. This indicates that the Evershed motion may be (at least partially) suppressed in the spine areas. Their continuum intensity is reduced mainly in the inner penumbra, while in the outer part the spines are less prominent in intensity.

\subsection{Azimuthal averages}

To investigate the mean properties of the two components, I averaged the field parameters azimuthally over ellipses with increasing radius. The results of the one-component fit are used in the umbra for the bg component. In Fig. 6, the results for the azimuthal averages of both observations ( 7 th and 9th) are shown for comparison. Starting in the lower right, the intensity in visible and IR continuum wavelengths has been used to place the transition from umbra to penumbra at $r / r_{\text {spot }}=0.4$, where $r_{\text {spot }}$ is the spot radius of around $13 \mathrm{Mm}$. The contrast of umbra and penumbra is greater for the visible wavelength range. The middle right panel shows that the relative filling fraction of the bg field and flow channels changes in the mid penumbra, the contribution of flow channels increases from $50 \%$ at $r / r_{\text {spot }}=0.65$ to a maximum of around $70 \%$ at $r / r_{\text {spot }}=0.8$. In the azimuthally averaged absolute LOS velocity, the observation at $30^{\circ}$ heliocentric angle shows a large difference of $2 \mathrm{~km} \mathrm{~s}^{-1}$ between bg component and flow channel, whereas the signature of the Evershed flow is negligible at $6^{\circ}$. In both cases the bg component shows increasing LOS velocities towards the outer boundary.

The field strength and field inclination of both observations are similar, indicating little change in the sunspot field topology in two days. In both observations, the bg component is stronger by $0.5 \mathrm{kG}$ in the inner and middle penumbra, whereas at the outer boundary the strength of both components is nearly identical. The slope with radius of the fc field strength decreases in the mid penumbra at $r / r_{\text {spot }}=0.7$ (dotted lines), but the field strength continues to drop with radius in both bg component and flow channels. The inclination of the fc component on average exceeds $90^{\circ}$ for $r / r_{\text {spot }}>0.9$, whereas the bg component never 

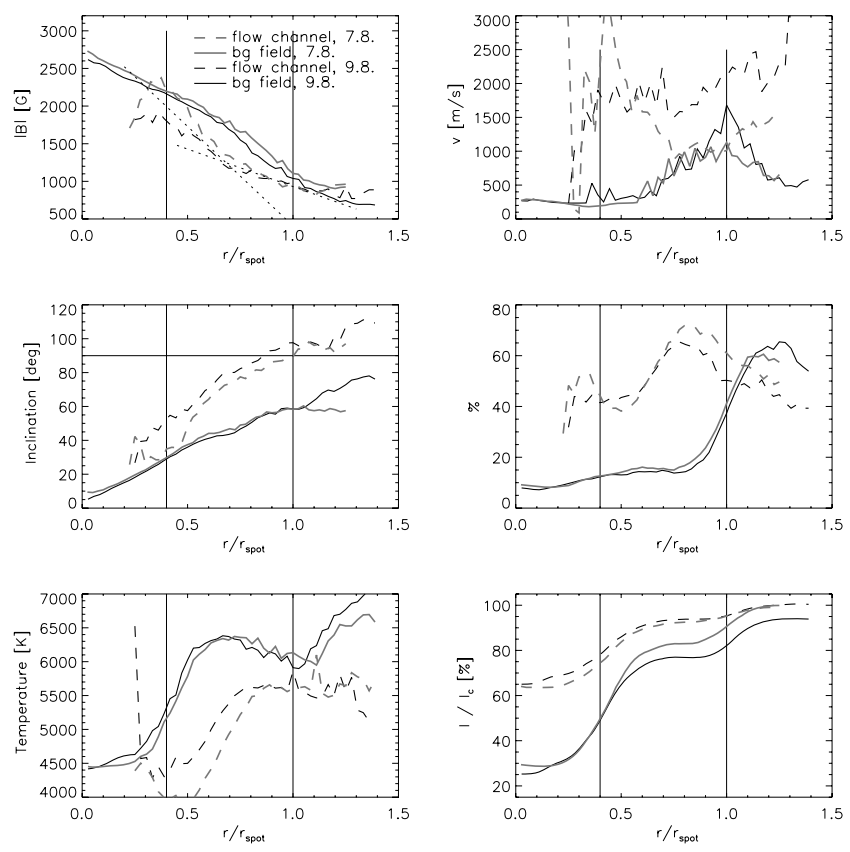

Fig. 6. Left column, top to bottom: radial variation of B, LRF inclination, temperature at $\log \tau=0$. Solid: bg component, dashed: fc component. Right column, top to bottom: averaged absolute LOS velocity; stray light amount (solid) with overplotted filling fraction of the fc component (dashed); intensity of visible (solid) and IR continuum (dashed). In this case, black (grey) lines refer to the observation on August 9 (7). The $x$-axis gives the fractional radial distance $r / r_{\text {spot }}$, the black vertical lines mark the inner and outer boundary of the penumbra. The horizontal black line in the inclination plot marks $90^{\circ}$.

turns into horizontal fields. Its maximum inclination at the outer penumbral boundary is close to $60^{\circ}$.

The temperature plot in the lower left panel shows that the radial variation of the temperature in the bg component follows the radial curve of intensity closely. The temperature curve of the fc component has a similar shape with reduced amplitude, but is displaced towards the outer penumbral boundary relative to the intensity or bg temperature curve. The decrease in the temperature in both components at the outer penumbral boundary is presumably connected to a trade-off between stray light and temperature in the inversion. At the outer boundary, the intensity level and shape of the profiles allows to use a larger stray light amount to reproduce the observed spectra, whereas in the umbra and penumbra the QS profile simply does not fit to the spectra. These results are in good agreement with the findings of Borrero et al. (2004) or Bellot Rubio et al. (2004), both in the absolute values of, e.g., field strength or field inclination and in their radial variation.

\subsection{Field-aligned flows}

With the assumption that on large spatial scales the flow velocities in the penumbra are only due to the radially aligned Evershed flow, the LOS velocity can be decomposed into its horizontal, $v_{\mathrm{h}}(r)$, and vertical component, $v_{z}(r)$ (e.g. Schlichenmaier $\&$ Schmidt 2000; Bellot Rubio et al. 2003, 2004). This allows to derive the flow angle, $\alpha(r)$; i.e., the inclination of the flow direction to the surface at a given radius. The flow angle can be derived separately for each inversion component, and then be directly compared to the average field inclination of the
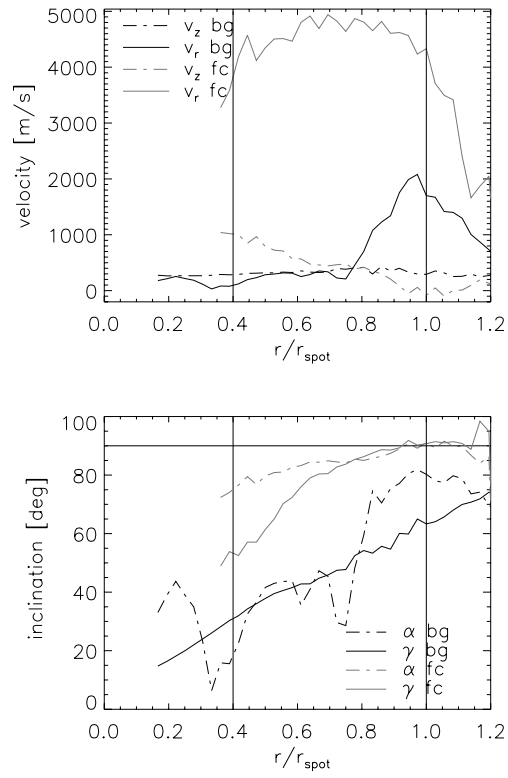

Fig. 7. Top: horizontal and vertical velocities for both inversion components. Black: bg component. Grey: fc component. Dashed: vertical velocity. Solid: horizontal velocity. Bottom: field inclination, $\gamma$ (solid), and flow angle, $\alpha$ (dashed).

component, $\gamma(r)$. Figure 7 displays the corresponding results for the observation on August 9. The fc component shows upflows of around $1 \mathrm{~km} \mathrm{~s}^{-1}\left(v_{z}>0\right)$ in the innermost penumbra, which turn into nearly horizontal flows with constant $4.6 \mathrm{~km} \mathrm{~s}^{-1}$ throughout the whole penumbra and finally exhibit a small downflow component for $r / r_{\text {spot }}>0.9$. The bg component shows a significant LOS velocity near the outer penumbral boundary, which could also be seen already in the azimuthal averages of Fig. 6. Bellot Rubio et al. (2004) point out that this non-zero velocity of the bg component is a necessary ingredient for correctly predicting the sign of the net circular polarization in the center side. The lower panel of Fig. 7 displays that the flows in the flow channels are parallel to the field inclination to a high degree in the outer penumbra. In the inner penumbra and for the bg component, the agreement is worse, but this may also be due to the intrinsic inaccuracies of the method. The azimuthal fine structure of dark and bright filaments is ignored by assuming that the absolute flow velocity $v \equiv v(r)$ only depends on radius. Given these limitations, I think that the result supports the concept of fieldaligned flows: upflows (downflows) are seen where the magnetic field inclination is below (above) $90^{\circ}$.

\subsection{Signature of hot upflows in the mid penumbra}

To investigate the radial structure of the fields without spatial averaging, I took the values of several quantities along a single column of the 2D maps at around $0^{\circ}$ field azimuth. Figure 8 shows this column and its surroundings. A hot upstream is located at about the middle of the penumbra. It has a strong signature in most quantities along the cut shown in Fig. 9. The LOS velocity in the fc component drops from $2 \mathrm{~km} \mathrm{~s}^{-1}$ farther inwards to slightly below zero and it takes around $2 \mathrm{Mm}$ to speed up again. The same is seen in the line-core velocity of Ti I. The inclination of the field is reduced from $88^{\circ}$ to $55^{\circ}$, but jumps to around $77^{\circ}$ on the next pixel $(\equiv 0.37)$ already. Rimmele (2004) and Rimmele \& Marino (2006) also find a rapid change of upflows 


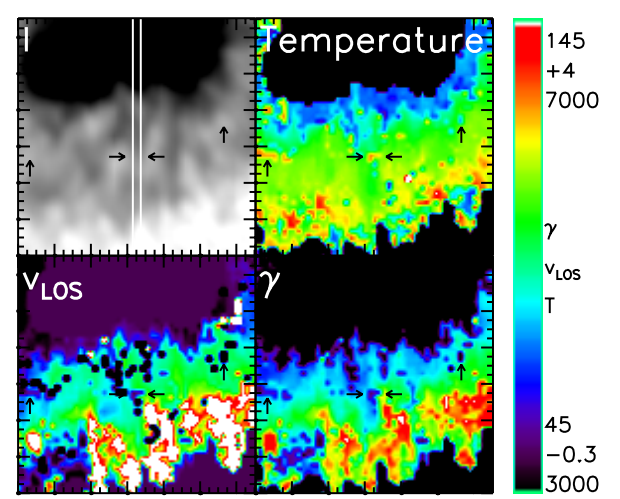

Fig. 8. Top row: continuum intensity and temperature of the fc component. Bottom row: velocity and inclination of the fc component. The white lines mark the column, along which the parameters shown in Fig. 9 were taken. Black arrows point towards hot upstreams in the mid penumbra.

into horizontal outflows on scales of around 0.' 6 . The temperature of the fc component increases by around $1000 \mathrm{~K}$ at the upstream location. In the continuum intensity, an increase is seen near the upstream, but displaced outwards by $1 \mathrm{Mm}$. Several similar hot upstreams in the mid penumbra with a change in temperature, flow velocity, and field inclination can be seen in Fig. 8; two examples near the left and right borders of the section shown are highlighted by arrows.

Significance of variation. The inversion procedure treats the spectra of each position independently of their surroundings. The inversion components are then separated with the two criteria (inclination, temperature) as described in Sect. 3. This can produce some strong pixel-to-pixel variations in the $2 \mathrm{D}$ maps of the inversion parameters. I think that, for the case shown in Fig. 9, I can exclude such an origin of the jump in atmospheric properties. The field inclinations differ significantly. The continuum intensities and the line-core LOS velocity of the Ti line are quantities that are not related to the inversion procedure, and they both also show the signature of a hot upstream: a local intensity maximum with a vanishing LOS velocity. Note that the situation is different from the "sea serpent" shape suggested by Schlichenmaier (2002), as I have no indications of downflows or field lines pointing down near the upstream. A possible configuration of the field lines using the integration of the inclination (cf. the next section) is shown in Appendix B, Fig. B.1.

\section{Derivation of the 3D field topology}

At first sight, the inversion with two magnetic components whose properties are constant with optical depth does not contain information on the vertical structure of the magnetic fields. Taking into account that the information on the field vector is available for an extended spatial region nonetheless allows the derivation of a 3D model by integrating the field inclination. The same method has already been proposed in Schlichenmaier $\&$ Schmidt (2000) and applied to the radial variation of the flow angle derived from spectroscopy. It has also been used in Solanki et al. (2003) and Wiegelmann et al. (2005) on measurements of the magnetic field vector. Solanki et al. (2003) used the requirement that the field azimuth did not change along the integration direction, which is fulfilled for the observed sunspot.

\subsection{Integration of field inclination}

For each radial position $r_{i}$, the azimuthally averaged inclination $\gamma\left(r_{i}\right)$ of bg component and fc component are known (cf. Fig. 6). With the assumption that the inclination does not strongly change with depth below the surface (or height above it), the geometrical height $h$ of a field line in the solar atmosphere can then be calculated from an integration of the inclination by

$h\left(r_{i}\right)=\sum_{j=0 \ldots i} \frac{1}{\tan \gamma\left(r_{j}\right)} \cdot \Delta r$,

where $\Delta r=330 \mathrm{~km}$ is the difference in the respective semimajor axes of two subsequent ellipses.

Two additional steps were applied to the curves of $h\left(r_{i}\right)$ for bg field and flow channels. The flow channels are not observed in the umbra, so no inclination values are available there. To have a common reference height in both integrated curves, I forced the height of bg and fc at $r / r_{\text {spot }}=1$ to be identical. This was motivated by the fact that spectra at the outer white-light boundary still showed the signatures of multiple components. Thus, both flow channels and bg component have to be present within the formation height of the spectral lines, hence, must be present at the same geometrical height as well. I also assumed that the isosurface of $\tau_{500}=1$ has a slope of $3 \mathrm{deg}$ when going from the inner towards the outer penumbral boundary (Schlichenmaier \& Schmidt 2000), corresponding to a Wilson depression of $380 \mathrm{~km}$ in the umbra. The Wilson depression agrees with the number given by Mathew et al. (2004), who however found no smooth radial change, but instead a pronounced jump of $280 \mathrm{~km}$ at the umbral-penumbral boundary. Figure 10 displays the finally resulting curves for both observations on August 7 and 9. Both observations yield very similar results.

The integrated inclination curve of the fc component yields a slightly elevated arched loop. The apex height above the $\tau=1$ level is around $300-400 \mathrm{~km}$. With the height fixed to $0 \mathrm{~km}$ at the spot radius, the curve intersects the $\tau=1$ level at around $r / r_{\text {spot }}=0.6$. Outside the sunspot, the field lines point downwards. As the bg component inclination never reaches horizontal fields, the integration yields a much steeper curve than for the flow channels. If taken at face value, the curve reaches a depth of 2 (4) Mm at $r / r_{\text {spot }}=0.8(0.6)$, implying a thick penumbra, not a shallow surface layer. For the bg component, fortunately a direct comparison with a theoretical model is possible. I overplotted the boundary layers between umbra and penumbra and between penumbra and surroundings from the magnetostatic sunspot model of Jahn \& Schmidt (1994, JS94) in Fig. 10. I reduced the radius in their original calculation to $93 \%$ and $95 \%$, respectively, of its value, to fit with the dimensions of the sunspot in the observations, and shifted the curves in height to be at $z=0 \mathrm{~km}$ at $r / r_{\text {spot }}=1$ like the integrated inclination curves. For $r / r_{\text {spot }}$ between 0.6 and 1 , the agreement between the integrated bg curve and the magnetostatic model is astonishingly good, whereas for smaller radii the JS94 model is steeper than the integrated curve.

The good agreement in the mid to outer penumbra comes a bit as a surprise: the JS94 model gives the location (and thus also the field inclination) of the boundary layer between spot and surroundings at depths up to some Mm, whereas the integrated curve uses the inclination observed at the surface close to the $\tau=1$ level. The observed surface inclination thus seems to be identical to the inclination in the deeper layers, which was simply an assumption in the derivation of the integrated curves. The deviation between the curves also points in the correct direction: if the inclination changes with depth, the fields should get more 

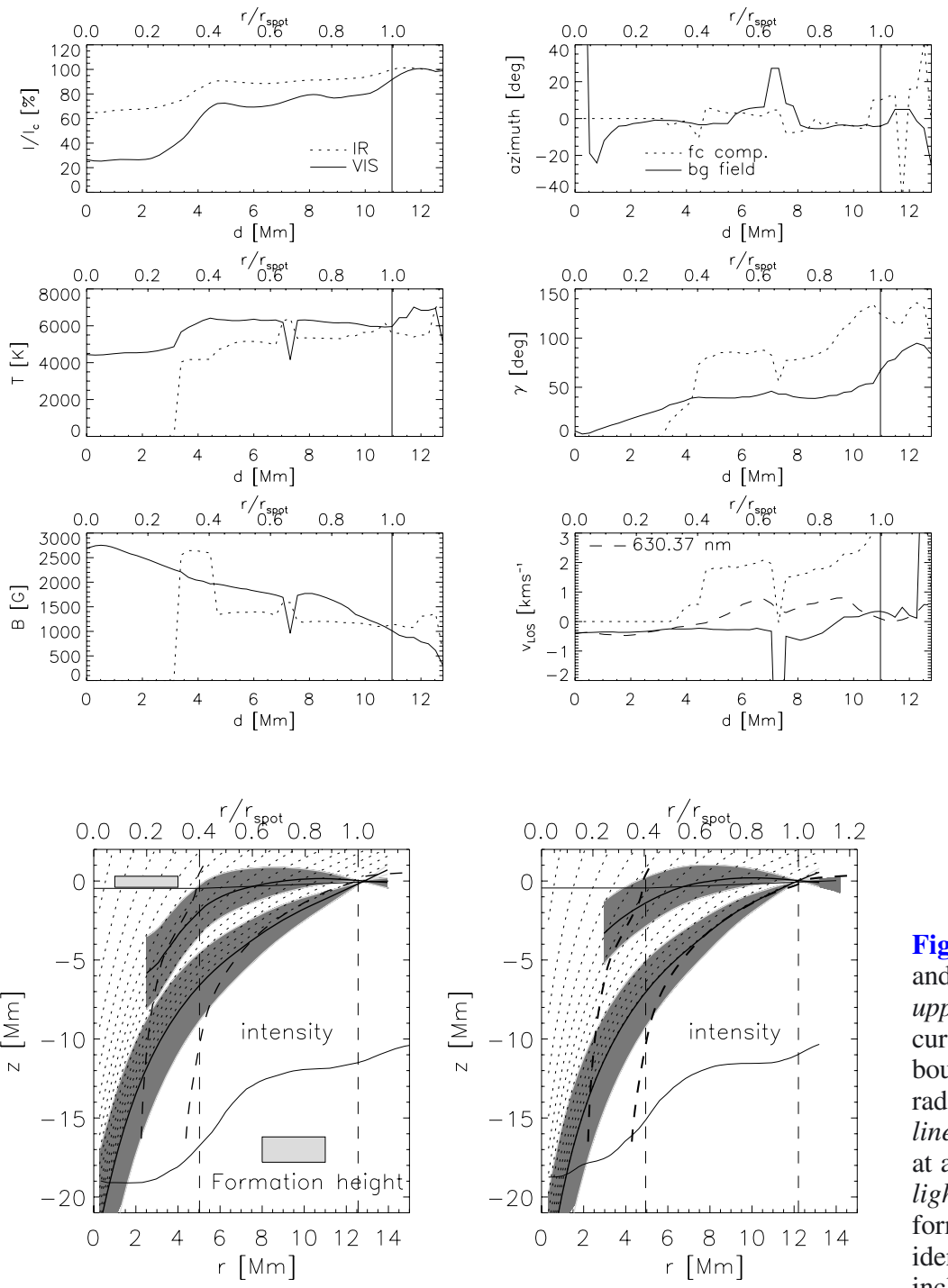

Fig. 9. Parameters along the column marked in Fig. 8. Left column, top to bottom: continuum intensity, temperature, field strength. Right column, top to bottom: field azimuth, field inclination, LOS velocity. In all graphs besides intensity, solid lines indicate quantities of the bg field and dotted lines those of the fc component. For intensity, solid and dotted correspond to visible, respectively, IR continuum intensity. The LOS velocity of TiI is given by the long-dashed line in the velocity graph. The vertical black line marks the outer penumbral boundary. The second hot upstream intersected by the cut at $d=6.2 \mathrm{Mm}$ has a similar signature in all quantities as the upflows at the inner penumbral boundary.

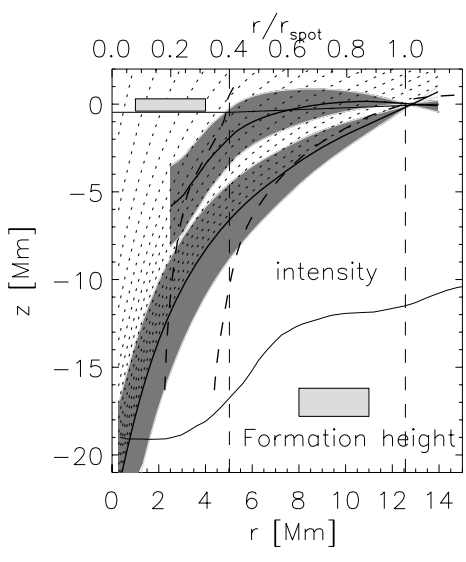

vertical in the deeper layers, as the expansion of flux concentrations happens near the surface layer. Thus, the surface inclination should be greater than in the deep layers, leading exactly to the shallower integrated curve as obtained.

Limitations of the integration. Despite the surprisingly good agreement with a theoretical model, which also indicates that the main assumption of a small depth dependence of the inclination could be valid, the integration of the surface inclination of course cannot be fully correct. In the left panel of Fig. 10, I overplotted the approximate formation height(s) of the observed spectral lines throughout the penumbra. All information retrieved from the observed spectra thus only refers to this small layer of the atmosphere. There is no guarantee that fields do not for example bend strongly as soon as they leave the height range, in which the spectral lines are sensitive. In fact, one expects exactly this behavior for the field lines that pass the upper boundary of the formation height, because of the exponential decrease in density with height. Sánchez Cuberes et al. (2005) found no height dependence of the field inclination using spectral lines similar to the $630.15 / 25 \mathrm{~nm}$ pair, but this would be valid only inside approximately the same formation height range as plotted in Fig. 10. To investigate the influence of inclination changes with height (or depth) on the integration of inclination, I repeated the integration with the assumptions that the inclination may vary with height by $\pm 20 \%$ for the bg component or respectively $\pm 15 \%$ for the flow channels. The upper and lower limits of the retrieved curves are given by shaded areas in Fig. 10. As the expected change should be a deviation towards more inclined fields in higher and less inclined fields in lower layers, any curve that would not leave the shaded areas could agree with the observed surface inclinations, within the $15-20 \%$ range of variation allowed for. Even if one takes the extreme case of a field line starting at the lower and ending at the upper boundary of the shaded area, the curves would not change enough to contradict the description above. The bg component still would imply a thick penumbra. The flow channels could change from nonelevated to strongly elevated loops, but without giving rise to a new topology of the flow channels relative to the bg component.

\subsection{A $3 D$ model}

In the previous section, the integration was performed for azimuthal averages, where the azimuthal fine structure of the penumbra is lost. The integration can, however, also be performed for individual radial cuts. To simplify the calculation and to smooth out the pixel-to-pixel variations slightly in the 

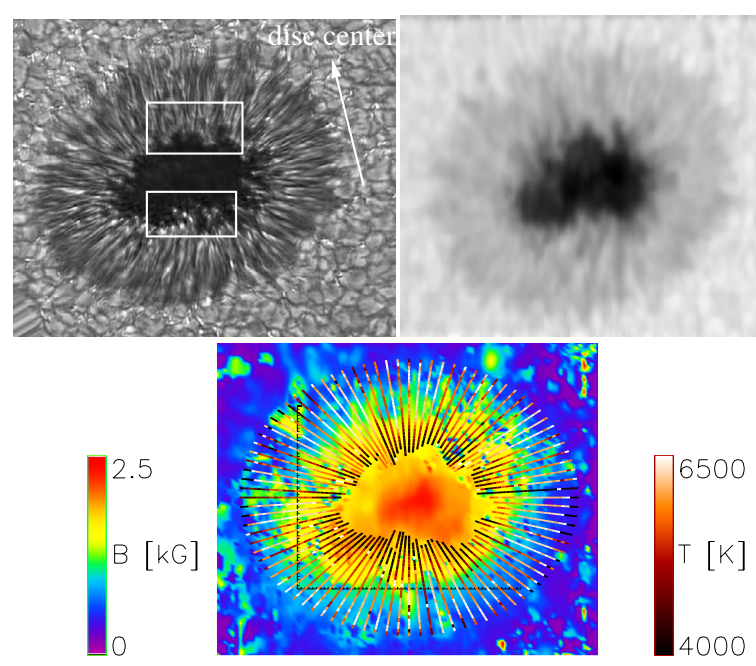

Fig. 11. Top left: image of NOAA 10425 in the G-band from the DOT on La Palma, taken on August 9 about half an hour after the observations at the VTT. The white arrow points towards disc center. The white rectangles mark regions with an identically structured umbra-penumbra boundary in both intensity maps. Top right: IR continuum map. Bottom: top view of the 3D model. See text for the description.

inversion results, I decided to use 92 bins $^{1}$ of about 4 deg angular extend for the construction of a 3D model that takes the azimuthal fine structure into account. The model itself is represented in the following way: the integration of the bg component gives a mesh of height with radial and azimuthal position, $h\left(r_{i}, \phi_{i}\right)$. This mesh is interpolated to a smooth surface, whose color is set to represent the field strength of the bg component. The flow channels are overplotted as thin lines, with a color code corresponding to their temperature. The temperature is scaled individually between the respective minimum and maximum for each of the 92 bins; thus, the color bar only gives an average range of temperature. This description applies to Figs. 11 and B.2.

As an intermediate step towards the full 3D model, Fig. 11 shows a top view of the model. This view corresponds to the commonly used 2D maps of physical quantities. For comparison, I also show a speckle-reconstructed image of NOAA 10425 in the G-band taken with the Dutch Open Telescope (DOT) on 2003 August 9 about half an hour later than the observation analyzed here. The figure serves as a comparison of the spatial resolution of the polarimetric to speckle-reconstructed data. The boundary between umbra and penumbra has the same shape in both observations (cf. inside the white rectangles), even with the time difference of half an hour. Bright penumbral grains can be seen in the DOT map near the umbral boundary, but only on the limb side.

Figure B. 2 shows the full 3D model for both observations for three different viewing directions ${ }^{2}$. I emphasize that the model is derived more or less straightforwardly from the observed spectra, with the single assumption that the integration of the field inclination is reasonable. If the model properties are projected to the surface at $z=0 \mathrm{~km}$, one has exactly the vector field and thermodynamic properties that gave the best fit to the observed Stokes profiles in - including the weak line blends -3 infrared and 4 visible spectral lines. The model(s) show some interesting

\footnotetext{
${ }_{1}^{1}$ It needed to be a multiple of 4 for technical reasons.

2 Two animations of the model for 2003 Aug. 9 are available in the online material.
}

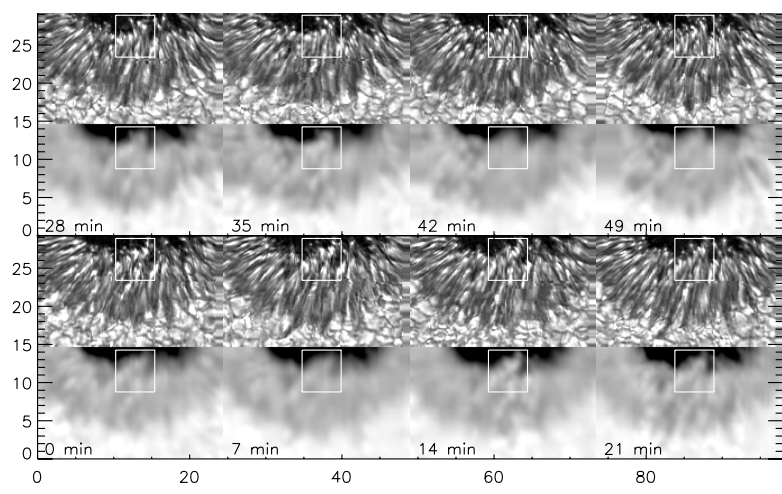

Fig. 12. The temporal evolution of the penumbra with 7 min cadence. Data of NOAA 10425 on 2003 August 9. The lower row shows the IR continuum intensity, the upper row the co-temporal and co-spatial map in the G-band from the DOT. Tick marks are arcsec; time increases from left to right in each row.

peculiarities and give rise to several interesting questions that could be addressed by analyzing them. The observation close to disc center (2003 Aug. 7, $6^{\circ}$ heliocentric angle) is very uniform all around the spot, whereas in the other case there is a much larger difference between the center and the limb side, where the flow channels appear to be much more elevated. The bg component exhibits a ragged subsurface structure, where less inclined and steeper fields coincide with an increased field strength. This could be closely related to the question if these "spines" (Lites et al. 1993) are co-spatial to darker or brighter filaments. In general, a study of the relation of intensity to this specific geometry would be interesting, because both positive and negative correlations between field strength, inclination, and intensity have been found up to now. Before an intense study of the question of the validity of the integration of inclination has been performed, I refrain from speculating further at this point. The 3D models show some similarity to the artist's sketch of a sunspot in Fig. 4 of Weiss et al. (2004), but I note that they are derived (directly) from observations here.

\section{Temporal evolution}

To investigate the temporal evolution, I used a time series taken later on August 9. Figure 12 shows a small subsection of the full field-of-view (FOV) at the DOT, together with a co-temporal observation taken at the VTT from 9:36 to 10:40 UT, after the map analyzed to derive the field topology. This is the upper part of the FOV containing the sunspot that was not used in Beck et al. (2007), where the data properties and alignment method are discussed in more detail. The images show a peculiarity of the penumbral dynamics that I think has not been given enough attention up to now. The global structure of the sunspot, e.g. the boundary between umbra and penumbra marked with a white rectangle, does not evolve at all in around $1 \mathrm{~h}$, whereas the intensity pattern of bright penumbral grains (PGs) evolves much faster on scales below 30 min (cf. also Appendix C). This suggests that the dynamical evolution does not change the geometry. In the context of the previous sections, this could be interpreted as a static bg component with a dynamic fc component.

\section{Discussion}

The penumbra has been the subject of many studies, which in most cases did not agree in their results. The observational 
findings are converging in some points, which I think I can substantiate further by the results of this investigation. The observations agree that the topology of the penumbra is complex. The Evershed flow happens along the nearly horizontal flow channels, whereas the average field inclination is not horizontal (e.g. Bellot Rubio et al. 2004). The Stokes $V$ profiles of Figs. 2 and 4 clearly show that at a resolution of 1 " at least two independent magnetic components are present in each pixel. This could be an artifact due to the spatial resolution, but if the picture of the uncombed penumbra is valid, it will be the case even if the flow channels were fully resolved. As long as the flow channels are not optically thick and are located inside the formation height of a spectral line, both horizontal and more vertical fields would be seen at the same time. Stokes spectra with higher spatial resolution from, e.g., the recently launched HINODE satellite could be used to see if the signature of multiple components disappears at high spatial resolution.

Some results turn out to be identical regardless of the inversion method employed, whether in a simple or complex model. If polarimetric profiles are analyzed in terms of two components (or also one component but with gradients, Borrero et al. 2004), one retrieves a stronger, less inclined component with slow flow velocities and a strongly inclined one, which harbors large flows. The flow is located inside the magnetic fields, as the flow velocity is derived from the Doppler shifts in the polarization signal. The same classification into a less inclined static field and more inclined flow channels was obtained by Langhans et al. (2005) from magnetograms. All recent inversions performed agree that the more inclined component bends downwards in the outer penumbra (Westendorp Plaza et al. 2001; Bellot Rubio et al. 2004; Borrero et al. 2004). Finally, hot upflows were found at the inner penumbral boundary in some other recent studies (Schlichenmaier et al. 2004; Tritschler et al. 2004; Borrero et al. 2005; Rimmele \& Marino 2006; Bellot Rubio et al. 2006).

With respect to the topology of the fields, I introduced the concept of integrating the surface inclination. This approach relies on the assumption that the field inclination does not depend on depth or height in the solar atmosphere, or only slightly does. Even if the validity of the assumption has not been addressed at all in this work, the method yields reasonable results. For the bg component, I find surprisingly good agreement of the integrated curve with the outer boundary layer between the sunspot and its surroundings in the magnetostatic model of JS94. This is especially unexpected, as their boundary line gives the field inclination in deep layers of some Mm, whereas I used the inclination on the surface. The integration of the fc inclination yields slightly elevated arched loops.

Another peculiarity refers to the behavior of the inversion results at around $r / r_{\text {spot }}=0.65$. At this location, the fill factor of the flow channels increases strongly. Westendorp Plaza et al. (2001) found a local maximum of intensity at $r / r_{\text {spot }}=0.6-0.7$; Bellot Rubio et al. (2004) found a change in the inversion results in the mid penumbra, a jump in the quantities of their more vertical component, and an increasing filling fraction of the fc component. Interestingly, an intersection point of the surface with the integrated curve is located near that radius. The inversion is performed pixel-by-pixel on only the spectra from a single location, whereas the integration uses the inclination results along a radial cut to create the curve of the field lines. As these two procedures are fully independent of each other, I think that this co-spatiality is not entirely pure coincidence. Taking the integrated curve at face value, the obvious explanation is that flow channels on average cross the surface around $r / r_{\text {spot }}=0.65$ and then have a stronger signature in the observed spectra.

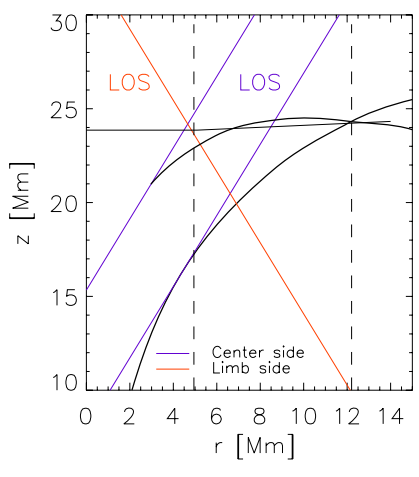

Fig. 13. The effects of the inclined LOS on observations of the limb or center side. Thick black: integrated LRF inclination of the 2-component inversion as in Fig. 10, fc component (upper line) and bg component (lower line). The LOS is overplotted in purple for the center side and in orange for the limb side.

\subsection{Line-of-sight effects}

The 3D models derived by the integration for different heliocentric angles show some deviations in their structure, even if azimuthally averaged values are nearly identical. The model of the observation near disc center is very uniform, while the other observation shows strong differences between limb and center side. As both observations were analyzed in exactly the same way, the difference should already be contained in the spectra. A second point related to LOS effects is the comparison with a high-resolution image of the same sunspot from the DOT telescope (cf. Fig. 11). Bright penumbral grains can only be seen on the limb side. This could be due to the simple geometrical effect depicted in Fig. 13. If the LOS is inclined to the surface, on the center side a limiting angle exists where a structure will be hidden by its own continuation. The two LOS lines on the center side in Fig. 13 intersect the bg and ft curves at locations where their inclination is the same as the heliocentric angle of the observation. The heliocentric angle of the observations on August 9 was around $30^{\circ}$, and the minimum field inclination of the fc component on the limb side was around $25^{\circ}$ (azimuthal average around $40^{\circ}$, cf. Fig. 6). Thus, the absence of the penumbral grains on the center side could be easily explained by the assumption that they are blocked by their own continuation as optically thick structures.

\subsection{The moving tube model}

$>$ From the observational point of view, a penumbral model of interlocked horizontal and more vertical fields, as given by the picture of the uncombed penumbra, is in good agreement with observed spectra, with their general shape down to more subtle details like the net circular polarization of Stokes $V$ profiles (Müller et al. 2006). From the various theoretical approaches, the moving tube model (MTM) of Schlichenmaier et al. (1998) is the only one that results by itself in a similar geometry, including a flow along the horizontal channels. The MTM uses the magnetostatic sunspot model of JS94 as input for the bg component. I find that either the integrated inclination curve, or, to be on the safe side, the observed inclination values of the bg field on the surface are in good agreement with the boundary layer between sunspot and surroundings in the JS94 model. However, the geometry of the background is not all that agrees with the observations. Figure 14 shows a direct comparison of the geometry, 


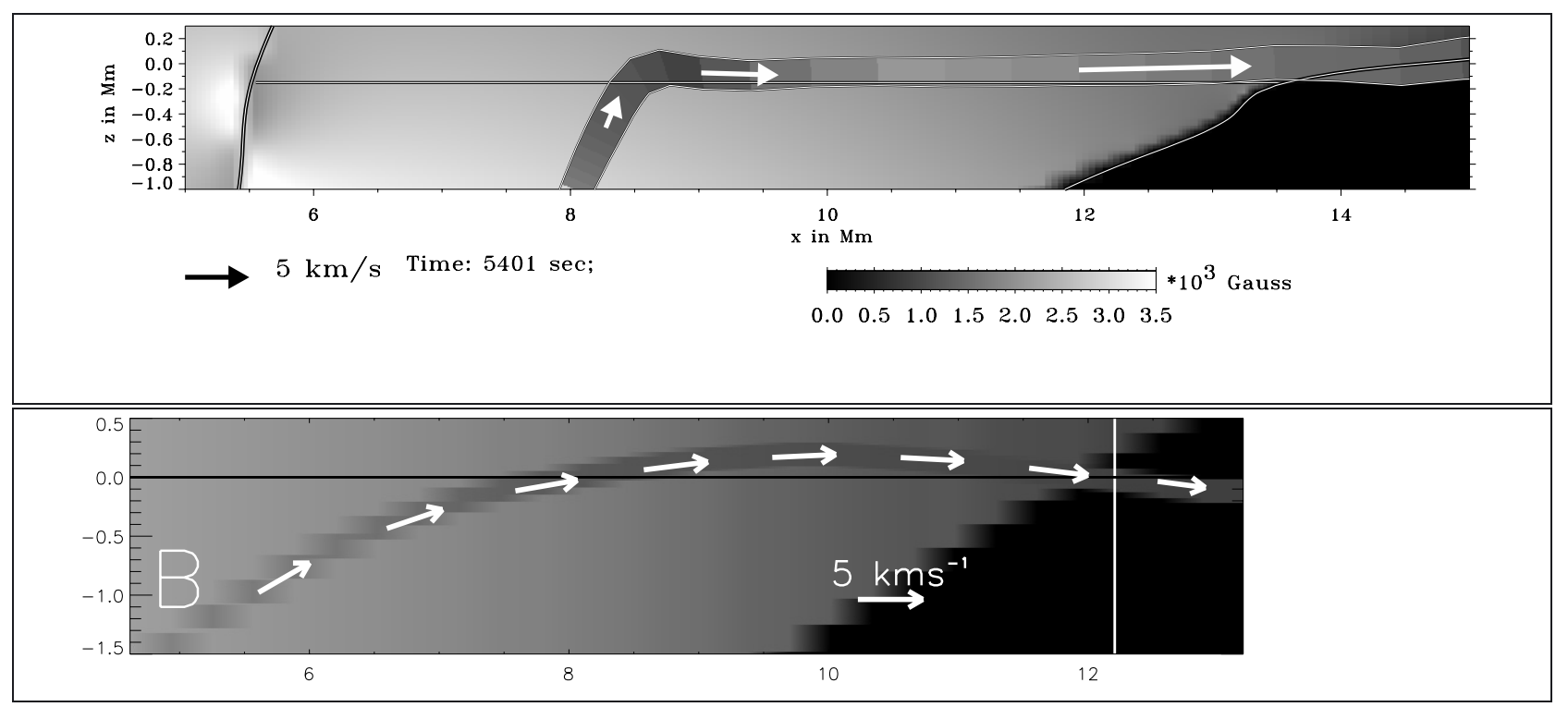

Fig. 14. Comparison of a snapshot of the moving tube model (top panel, courtesy of R. Schlichenmaier) with the flow channels' geometry from the observation (lower panel). The plots have been arranged to have the same scaling and position in the horizontal axis that gives the distance from the spot center in $\mathrm{Mm}$. Both panels display the fc component and the bg component with their respective field strength as color coding. The color bar gives the color coding for the field strength in both graphs. Velocities are overplotted as arrows, whose length gives the absolute velocity. The direction of the arrows for the lower plot is the field inclination.

field strength, and absolute velocity of a snapshot of the MTM during the final steady-state of the simulations and the same result as derived from the observations. For the location of the flow channel, I took the value of the integrated inclination curve and the integrated bg inclination curve for the outer sunspot boundary. The flow velocity and the field strength in the flow channel and bg component were taken from the azimuthal averages of the inversion results. The only quantity that was defined ad hoc was the width of the flow channels, on which the present inversion does not yield any information; I used a diameter of $200 \mathrm{~km}$ for it (cf. Beck 2006). I find quite some similarity between the MTM and the results derived from the observations. Both field topology and the local properties (field strength, flow velocities in the inner and mid penumbra) do match reasonably well. Note that from the theoretical side the differences between the MTM and the integrated curve in, e.g., the height of the flow channel ( 100-200 km) will lead people to argue that the curve suggested by the integration is inconsistent with any physics. I must, however, point out that it reflects the azimuthal average of, at maximum, half-resolved flow channels converted to a geometric height without much sophistication, whereas the MTM is supposed to describe an individual resolved structure. Spruit \& Scharmer (2006) and Scharmer \& Spruit (2006) have raised the issue of the stability of the flow channels of assumed circular diameter inside the penumbra, and claim that no stable configurations are possible. The present investigation using model components without depth variation of the field properties actually does not yield information on this topic, but the inversion results and the integration of the inclination with the assumption of no depth variation would still apply for the case of flow sheets with a small lateral and large vertical extension. Finally, the agreement of the NCP predicted by the MTM and the observations has already been partly discussed in Müller et al. (2006). A more detailed comparison of observed NCP, the NCP predicted from the MTM, and the NCP resulting from a fit of an uncombed penumbral model to the same observations used here is planned to appear in another paper.

\subsection{The "gappy" penumbra model}

Spruit \& Scharmer (2006) and Scharmer \& Spruit (2006) suggest a model of field-free gaps as a possible mechanism of the penumbral energy transport. Even if a direct comparison of their model with observations is difficult, as yet no (polarization) spectra have been presented for their model, some of their arguments can be compared to the present findings. Spruit \& Scharmer (2006) suggested that the amount of stray light found in inversions could be related to the presence of a fieldfree component. The stray light amount in the inversion of the present observations increases smoothly from around $10 \%$ in the umbra through the penumbra $(\sim 10-15 \%)$ to $80 \%$ at the outer penumbral boundary. The $2 \mathrm{D}$ map of the stray light contribution (cf. Fig. 5) shows little spatial structure besides the radial increase. The stray light inside the telescope and the instruments TIP and POLIS was estimated to be around 15\% (Rezaei et al. 2007; Cabrera Solana et al. 2007). There thus is little indication for a field-free component inside of the sunspot in the inversion results.

Scharmer \& Spruit (2006) argue that the inversion of polarimetric data has a high degree of ambiguity, as similar profiles can result from different atmosphere stratifications. Using the IR lines at $1.5 \mu \mathrm{m}$, I think that most of the ambiguities are removed. In Beck (2006) and Beck et al. (2007) I investigated the influence of the spectral lines on the parameters retrieved by the inversion. I found that e.g. field strength is restricted by the splitting of the $1564.8 \mathrm{~nm}$ line within a limit of around $\pm 100 \mathrm{G}$. Most average quantities of the field topology (field strength, field orientation) are more or less uniquely restricted by the spectra, with the main source of error actually the spectra themselves: spatial resolution, signal-to-noise ratio, polarimetric sensitivity, and the polarimetric calibration. The question that remains open, however, is the 3D organization of the magnetic fields. The present inversion yields field strength and orientation inside the formation height of the spectral lines, but is not able to differentiate between a vertical or horizontal interweavement of field lines. 
The approach of the integration of the field inclination assumes coherent structures from one pixel to the next in the radial direction, which is highly probable in my opinion, but need not be the case.

The most prominent spectral feature inside the penumbra, the Evershed effect, is not present in the gappy model. I note that all velocities derived here (besides the line-core velocity of Ti I) always refer to velocities inside magnetic fields. To create the multi-lobed profiles in the neutral line of Stokes $V$, two components of magnetic fields with different orientations and bulk velocities are needed. If the gappy model solves the penumbral heat transport problems, an explanation for the Evershed flow is still needed. The inclination of the bg field shows an azimuthal variation that leads to a "gappy" structure (cf. Fig. B.2). However, the spatial scale of the variation is larger than predicted by Scharmer $\&$ Spruit (2006). If the integrated curves are taken at face value, this also happens in layers far below the surface layer of $\tau=1$.

Another argument in favor of the gappy model is that the spatial resolution of the observations I used may not be high enough to detect the signatures of the structuring suggested by Scharmer \& Spruit. In the other direction, it should be possible to construct a sunspot model based on their suggestions, calculate the resulting spectra in the $1.5 \mu \mathrm{m}$ and $630 \mathrm{~nm}$ lines, reduce the spatial resolution to around $1^{\prime \prime}$, and then invert the spectra with two depth-independent magnetic components. The results for the bg component could be compared with the present inversion results. Contrary to the regrettable sentence of Scharmer \& Spruit (2006) that "the agreement with observations obtained with such [uncombed] models is of unquantifiable significance", I think it necessary to show that their model successfully reproduces spectroscopic or spectropolarimetric observations to support its validity.

\subsection{A model for the penumbral energy transport}

The long lifetimes (on the order of $1 \mathrm{~h}$ ) for filaments (e.g. Langhans et al. 2005) and the lack of submerging flow channels led Schlichenmaier \& Solanki (2003) to the conclusion that interchange convection by rising hot flow channels is "not a viable heating mechanism" for the penumbra. This claim has been renewed recently by Spruit \& Scharmer (2006) and Scharmer \& Spruit (2006), who criticize the "paradigm" of embedded flow channels and suggest that the penumbral fine structure can be explained by a model of field-free gaps reaching almost up to the solar surface. The energy transport in their model is then achieved by convection in the field-free plasma below the sunspot.

On the one hand, the findings of Sect. 6 support the static behavior of the sunspot fields: the shape of the umbral-penumbral boundary and some especially dark patches inside the penumbra stay the same during around $1 \mathrm{~h}$. On the other hand, the intensity pattern of, e.g., the penumbral grains is completely changed after less than half an hour (cf. Appendix C). The time scales in the MTM model are of a comparable order. The snapshot shown in Fig. 14 was taken at $11 / 2 \mathrm{~h}$ after the start of the simulation and reflects the final steady-state solution. The flow channel in the MTM evolves rapidly in the beginning and spans around half of the penumbra after $30 \mathrm{~min}$ (Schlichenmaier et al. 1998).

Langhans et al. (2005) used LOS magnetograms of the center side penumbra. These LOS magnetograms are not suitable for tracing the dynamic evolution of the penumbra. On the center side, the field of the bg component is parallel to the line of sight, whereas the dynamic flow channels are strongly inclined to it. Thus, the lifetimes measured there reflect the slow evolution of the bg component. The same applies to the findings of Sect. 6: the stronger field component will dominate the topology, and thus, the small amount of change in the geometry seen in Fig. 12 implies that the background component does evolve but only slowly.

The conclusion about the impossibility of interchange convection due to the lack of submerging flow channels needs some more explanations. To be convinced by the argument, one would have to agree that the penumbra is deep (some $\mathrm{Mm}$ ), that flow channels originate from magnetic flux located initially on the boundary layer between the sunspot and its surroundings, and that this magnetic flux can become buoyant by heat input from the fully convective surroundings outside the $\operatorname{spot}^{3}$. The first point is suggested by the observations, while the last two are mainly based on the MTM. If one can agree on the ingredients above, I believe the penumbral heat transport can be achieved by hot rising flow channels in full agreement with the observations. If magnetic flux becomes buoyant at the outer sunspot boundary due to heating, this is necessarily a repetitive process. As soon as the hot flux bundle has risen from the boundary layer, new different magnetic flux will form the boundary layer. This new magnetic flux would come from the bg component, in the terminology used throughout this paper. After a time span on the order of $30 \mathrm{~min}$ it would also have to become buoyant, and follow the previously risen magnetic flux upwards. Due to the depth of the penumbra, several flow channels could be stacked on top of each other at the same time. The inversion results along a single column (cf. Sect. 4.4 and Appendix B.1) suggest exactly this configuration: two flow channels are seen along a radial cut at the same time at different locations in the penumbra.

The question of penumbral heating then changes to the question of whether the penumbral energy losses can be replenished by more than one hot flow channel with a characteristic repetition time around $30 \mathrm{~min}$. It has been shown by Schlichenmaier et al. (1999) and Schlichenmaier \& Solanki (2003) that the final state of the MTM is not able to supply the penumbral heat requirements. The final state of the MTM is also, however, not in agreement with the temporal evolution of penumbral fine structure. Penumbral grains do not persist for hours, but fade away after reaching the umbral boundary, or turn into umbral dots without a visible connection to the penumbra (e.g. Sobotka et al. 1995). The MTM never reaches this point, probably due to its boundary condition, which fixes the lower foot point of the flow channel to the outer boundary layer between spot and surroundings. If the source of heat input is the convective surroundings outside the spot, it is easily conceivable that a flow channel would start to disappear, if it detaches completely from the boundary layer.

The repeated ascent of flow channels of course would on the long term move all magnetic flux away from the boundary layer, leading to the disappearance of the penumbra after a short time. To replace the magnetic flux, no submerging flow channels are needed. The replacement of the boundary layer can be achieved by a gradual re-arrangement of the magnetic flux of the bg component. The open question then is whether a flow channel can become part of the bg component after reaching the umbra. This should not be impossible: the inclination difference between flow channels and bg component in the innermost penumbra is only around $20^{\circ}$ on average. Furthermore, the inner, more vertical foot point of the flow channel will contribute to the magnetic field pressure term in the inner penumbra,

${ }^{3}$ I guess, less than half of the solar physicists will agree on that. 


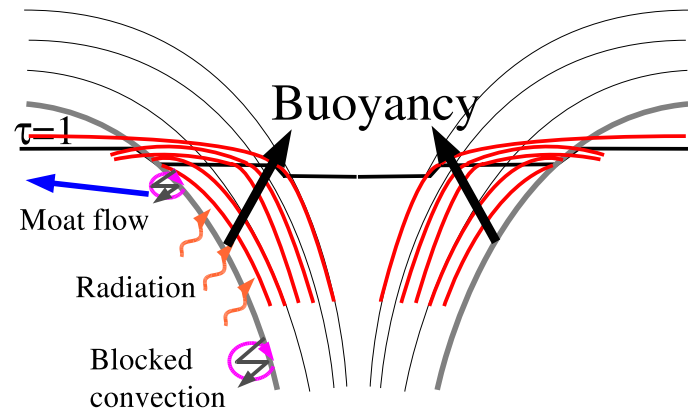

Fig. 15. Schematical model of the sunspot structure.

pushing other field lines of the bg field radially outwards towards the magnetopause.

\section{Summary and conclusions}

I have analyzed two spectropolarimetric observations of NOAA 10425 , taken two days apart, at heliocentric angles of $6^{\circ}$ and $30^{\circ}$, respectively. The observations consisted of Stokes vector polarimetry of four visible spectral lines around $630 \mathrm{~nm}$ and three infrared spectral lines at $1.5 \mu \mathrm{m}$, taken with POLIS and TIP. I inverted the spectra with the SIR code, using two independent magnetic model components. The field properties were assumed to be constant with optical depth. I sorted the two magnetic components by their inclination to the surface normal and refer to the more (less) inclined component as flow channels (background component). In the innermost penumbra, the inclinations of the two components were nearly identical, and I used the temperature as the criterion instead.

The inversions of the same spot on the two days agree that the more inclined component is weaker by around $0.5 \mathrm{kG}$ in the innermost penumbra, but it has the same strength as the more vertical bg component at the outer spot boundary due to less of a decrease in field strength with distance from the spot center, as also found by Borrero et al. (2004) or Bellot Rubio et al. (2004). I find hot upstreams in the mid penumbra, and they appear in the more inclined component. The hot upflows show, however, more vertical fields in the fc component than in neighboring pixels. The more inclined field component shows high flow velocities up to $5 \mathrm{~km} \mathrm{~s}^{-1}$ throughout the whole penumbra, which I find to be roughly aligned with the field direction. At the outer spot boundary, the flow channels on average bend slightly downwards to return to the surface (or submerge below it), whereas the bg component never exceeds an inclination of $60-70^{\circ}$. Both inverted sunspot maps show a change in the relative filling fraction of bg component and fc component at around $r / r_{\text {spot }}=0.65$, where the filling fraction of the fc component increases.

To generate a geometrical model of the penumbral field topology, I integrated the surface inclination in the radial direction. This approach is valid, if the field inclination (not field strength!) is independent of or only slightly dependent on depth in the solar atmosphere. This method can, on the one hand, be used as a powerful visualization tool, as it allows the geometry and all other field properties to be easily set in context. On the other hand, separately integrating the inclination of bg component and fc component, it allows construction of a 3D model of the sunspot that is basically the best-fit atmosphere model for the observed profiles. In this 3D model, the bg component shows steeper ridges of enhanced field strength, which may be identical to the "spines" of Lites et al. (1993). The azimuthally averaged fc component yields arched loops that cross the $\tau=1$ surface at around $r / r_{\text {spot }}=0.65$, where the filling fraction changes in favor of the flow channels. There is no obvious reason why these two things happen at the same location, because the inversion is done pixel-by-pixel without any information from neighboring pixels, whereas the integration uses all inclination values in the radial direction. I thus conclude that it is highly probable that on average the flow channels do cross the surface at this radius.

In general, the inversion results agree with the simulations of the moving tube model of Schlichenmaier et al. (1998) in several aspects (geometry of bg component and flow channels, radial variation of physical quantities). With a characteristic penumbral time scale of intensity variations below $30 \mathrm{~min}$ and a depth of the penumbra of some Mm as suggested by the integrated inclination, I think that nothing found in the present observations would contradict a penumbral heat transport by a series of hot, consecutively rising flow channels, as depicted in Fig. 15. An analysis of a time series of sunspot observations using a twocomponent inversion and the integration of the field inclination may be able to give more direct proof if this scenario is actually happening in the penumbra. I will attempt this task as the next step using either the data shown in Fig. 12 or observations from later times that were taken with the help of an adaptive optics system (e.g., Cabrera Solana et al. 2006).

Acknowledgements. The VTT is operated by the Kiepenheuer-Institut für Sonnenphysik (KIS) at the Spanish Observatorio del Teide of the Instituto de Astrofísica de Canarias (IAC). The DOT is operated by Utrecht University at the Spanish Observatorio del Roque de los Muchachos of the IAC. This work has been partly supported by the Deutsche Forschungsgemeinschaft under grant SCHL 512/2-1. The POLIS instrument is a joint development of the High Altitude Observatory (Boulder, USA) and the KIS. Discussions and advice from L. R. Bellot Rubio, R. Schlichenmaier, and especially the supervisor of my thesis at the KIS, W. Schmidt, are gratefully acknowledged. I thank the referee for pointing out the problems with the identification of the two components in the inner penumbra.

\section{References}

Ballesteros, E., Collados, M., Bonet, J. A., et al. 1996, A\&AS, 115, 353 Beck, C. 2006, Ph.D. Thesis, Albert-Ludwigs-University, Freiburg Beck, C., Schlichenmaier, R., Collados, M., Bellot Rubio, L., \& Kentischer, T. 2005a, A\&A, 443, 1047

Beck, C., Schmidt, W., Kentischer, T., \& Elmore, D. 2005b, A\&A, 437, 1159

Beck, C., Bellot Rubio, L. R., Schlichenmaier, R., \& Sütterlin, P. 2007, A\&A, 472, 607

Bellot Rubio, L. R., Collados, M., Ruiz Cobo, B., \& Rodríguez Hidalgo, I. 2000, ApJ, 534, 989

Bellot Rubio, L. R., Balthasar, H., Collados, M., \& Schlichenmaier, R. 2003, A\&A, 403, L47

Bellot Rubio, L. R., Balthasar, H., \& Collados, M. 2004, A\&A, 427, 319

Bellot Rubio, L. R., Schlichenmaier, R., \& Tritschler, A. 2006, A\&A, 453, 1117 Borrero, J. M., \& Bellot Rubio, L. R. 2002, A\&A, 385, 1056

Borrero, J. M., Solanki, S. K., Bellot Rubio, L. R., Lagg, A., \& Mathew, S. K. 2004, A\&A, 422, 1093

Borrero, J. M., Lagg, A., Solanki, S. K., \& Collados, M. 2005, A\&A, 436, 333

Cabrera Solana, D., Bellot Rubio, L. R., \& del Toro Iniesta, J. C. 2005, A\&A, 439, 687

Cabrera Solana, D., Bellot Rubio, L. R., Beck, C., \& del Toro Iniesta, J. C. 2006, ApJ, 649, L41

Cabrera Solana, D., Bellot Rubio, L. R., Beck, C., \& del Toro Iniesta, J. C. 2007, A\&A, 475, 1067

Collados, M. 2002, Astron. Nachr., 323, 254

Evershed, J. 1909, MNRAS, 69, 454

Galilei, G. 1632, Dialogo dei massimi sistemi, Fiorenza, Per Gio: Batista Landini Galilei, G., Welser, M., \& de Filiis, A. 1613, Istoria e dimostrazioni intorno alle macchie solari (Roma: G. Mascadi)

Gingerich, O., Noyes, R. W., Kalkofen, W., \& Cuny, Y. 1971, Sol. Phys., 18, 347 Hale, G. E. 1908, ApJ, 28, 315

Jahn, K., \& Schmidt, H. U. 1994, A\&A, 290, 295

Langhans, K., Scharmer, G. B., Kiselman, D., Löfdahl, M. G., \& Berger, T. E. 2005, A\&A, 436, 1087

Lites, B. W., Elmore, D. F., Seagraves, P., \& Skumanich, A. P. 1993, ApJ, 418, 928 
Müller, D. A. N., Schlichenmaier, R., Steiner, O., \& Stix, M. 2002, A\&A, 393, 305

Martínez Pillet, V. 2000, A\&A, 361, 734

Martínez Pillet, V., Collados, M., Sánchez Almeida, J., et al. 1999, in High Resolution Solar Physics: Theory, Observations, and Techniques, ASP Conf. Ser., 183, 264

Mathew, S. K., Solanki, S. K., Lagg, A., et al. 2004, A\&A, 422, 693

Müller, D., Schlichenmaier, R., Fritz, G., \& Beck, C. 2006, A\&A, 460, 925

Nave, G., Johansson, S., Learner, R. C. M., Thorne, A. P., \& Brault, J. W. 1994, ApJS, 94, 221

Rezaei, R., Schlichenmaier, R., Beck, C., Bruls, J., \& Schmidt, W. 2007, A\&A, 466, 1131

Rimmele, T., \& Marino, J. 2006, ApJ, 646, 593

Rimmele, T. R. 2004, ApJ, 604, 906

Ruiz Cobo, B. 1998, Ap\&SS, 263, 331

Ruiz Cobo, B., \& del Toro Iniesta, J. C. 1992, ApJ, 398, 375

Sánchez Almeida, J., \& Lites, B. W. 1992, ApJ, 398, 359

Sánchez Cuberes, M., Puschmann, K. G., \& Wiehr, E. 2005, A\&A, 440, 345

Scharmer, G., \& Spruit, H. 2006, A\&A, 460, 605

Schlichenmaier, R. 2002, Astron. Nachr., 323, 303

Schlichenmaier, R., \& Collados, M. 2002, A\&A, 381, 668

Schlichenmaier, R., \& Schmidt, W. 2000, A\&A, 358, 1122
Schlichenmaier, R., \& Solanki, S. K. 2003, A\&A, 411, 257

Schlichenmaier, R., Jahn, K., \& Schmidt, H. U. 1998, A\&A, 337, 897

Schlichenmaier, R., Bruls, J. H. M. J., \& Schüssler, M. 1999, A\&A, 349, 961

Schlichenmaier, R., Bellot Rubio, L. R., \& Tritschler, A. 2004, A\&A, 415, 731

Schmidt, W., \& Kentischer, T. 1995, A\&AS, 113, 363

Sobotka, M., Bonet, J. A., Vazquez, M., \& Hanslmeier, A. 1995, ApJ, 447, L133+

Solanki, S. K., \& Montavon, C. A. P. 1993, A\&A, 275, 283

Solanki, S. K., Lagg, A., Woch, J., Krupp, N., \& Collados, M. 2003, Nature, 425, 692

Spruit, H., \& Scharmer, G. 2006, A\&A, 447, 343

Thomas, J. H., \& Weiss, N. O. 2004, ARA\&A, 42, 517

Tritschler, A., Schlichenmaier, R., Bellot Rubio, L. R., et al. 2004, A\&A, 415, 717

Weiss, N. O., Thomas, J. H., Brummell, N. H., \& Tobias, S. M. 2004, ApJ, 600, 1073

Westendorp Plaza, C., del Toro Iniesta, J. C., Ruiz Cobo, B., et al. 2001, ApJ, 547,1130

Wiegelmann, T., Lagg, A., Solanki, S. K., Inhester, B., \& Woch, J. 2005, A\&A, 433, 701

Wittmann, A. D., \& Xu, Z. T. 1987, A\&AS, 70, 83 

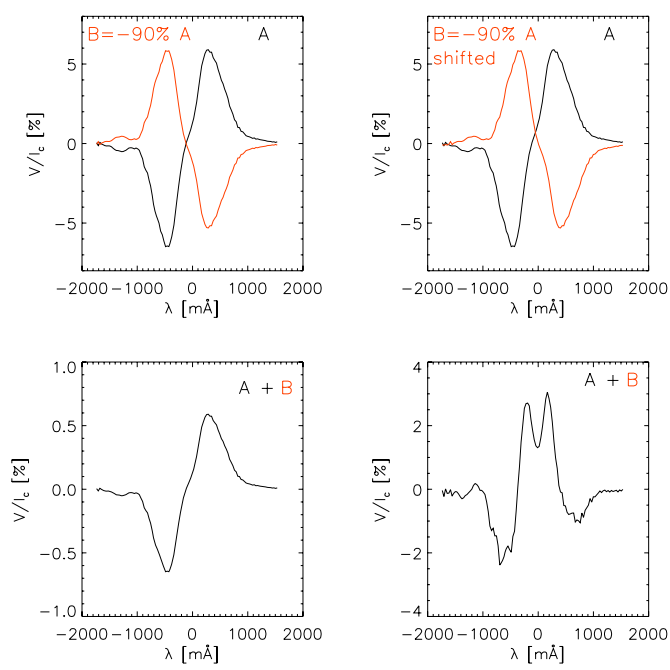

Fig. A.1. A simple method of producing peculiar $V$ profiles. The addition of the two profiles A and B in the top row yields the profiles in the bottom row. Left column: profile B equals $-90 \%$ of A and is located at the same wavelength. Right column: B equals $-90 \%$ of A, but now shifted in wavelength.

\section{Appendix A: Creation of multi-lobed profiles}

The Stokes $V$ profiles in the neutral line on the limb side (Fig. 2) show a complex multi-lobed structure. Although the profiles strongly differ from those originating from a simple atmosphere with constant magnetic field properties in the formation height of the spectral lines, only a few ingredients are needed to reproduce their shapes to first order. Figure A.1 shows a simple experiment: the regular profile from the center side (rightmost graph of Fig. 2) is taken to be component A; a second component $\mathrm{B}$ is constructed as $-90 \%$ of $\mathrm{A}$. Adding these two profiles again generates a regular two-lobed profile with reduced amplitude (left column of Fig. A.1). If component B is displaced in wavelength - corresponding to the Doppler shift induced by a flow field of $2 \mathrm{~km} \mathrm{~s}^{-1}$ - the addition yields peculiar multi-lobed profiles like those observed in the neutral line of Stokes $V$.

\section{Appendix B: 2/3D models}

\section{B.1. 2D: integration of single column}

To follow the properties in a spatial cut without averaging, I took the inversion results along a single column of the 2D maps (cf. Sect. 4.4). The inclination of the cut can also be integrated in the radial direction. In Fig. B.1, I display the result in the same way as in Fig. 14, but this time with the temperature as color coding. The hot upstream is located at $x=9 \mathrm{Mm}$. The integrated curve was corrected to meet $z=0 \mathrm{~km}$ at the outer sunspot boundary. Then I suggestively have shifted the integrated curve for $x>9 \mathrm{Mm}$ down by $150 \mathrm{~km}$ to meet the $\tau=1$ level at $x=9 \mathrm{Mm}$. As the formation height covers some hundred kilometers, the geometry depicted would still agree with the observed spectra, even if of course I have no proof that the second channel is below the first one.

\section{B.2. $3 D$ models}

Figure B. 2 shows the full 3D model for both observations from three different viewing directions: from above, the side, and

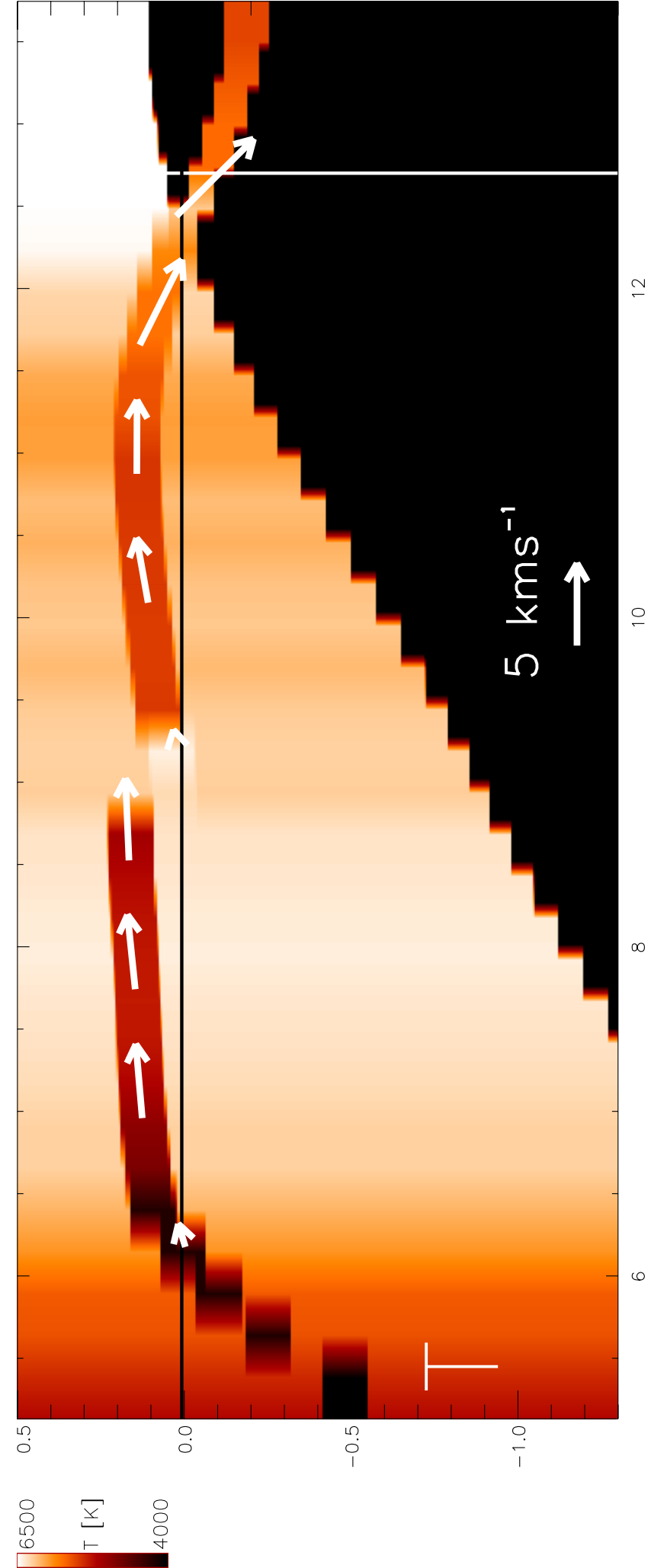

Fig. B.1. The integrated inclination values along the single column marked in Fig. 8. Similar to Fig. 14, but using temperature for the color coding. The integrated curve has been shifted down at $x=7 \mathrm{Mm}$, where the hot upstream appears.

slightly below the surface. The color code and display method correspond to that of Fig. 11. Two animations showing the 

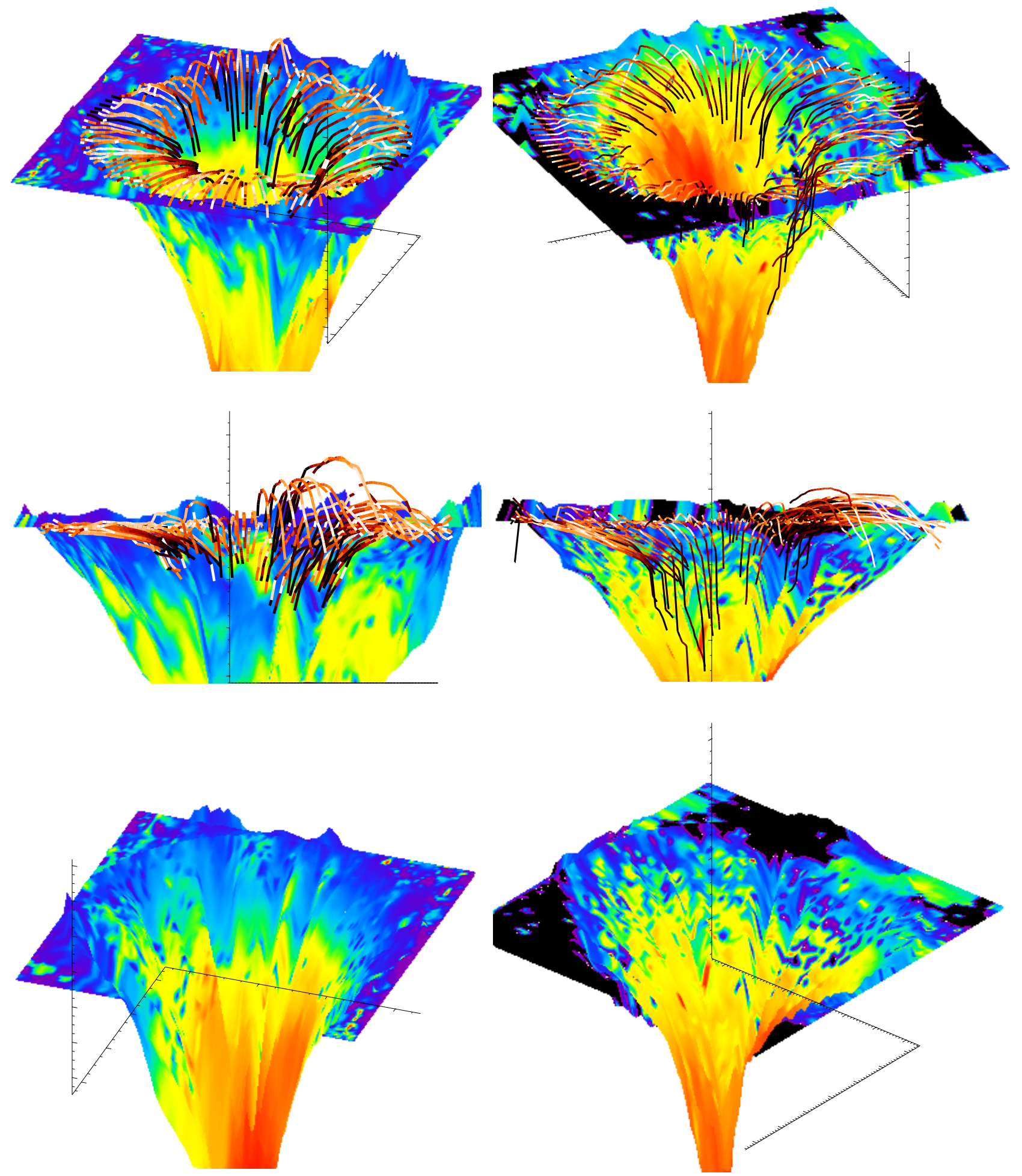

Fig. B.2. The 3D models from the integration of the inclinations to the surface averaged over 4 degree bins. Left column: data of August 9. Right column: data of August 7. Top to bottom: view from above, side view, view from below. The color coding is identical to Fig. 11 . The plot of the flow channels was switched off for the view from below.

sunspot model of 2003 Aug. 9 are available in the online material.

\section{Appendix C: Analysis of DOT time series}

To quantify the amount of dynamics in the penumbra, I used a 2-h time series of speckle-reconstructed images in the G-band from the DOT telescope, taken on 2003 August 9 after the map used for the present investigation $\left(30^{\circ}\right.$ heliocentric angle). To derive the boundaries of the penumbra, I used the temporal average of the time series (cf. Fig. C.1). To avoid counting brightenings at the outer white-light boundary as penumbral grains (PGs), I further restricted the area to all points inside an ellipse centered on the spot, but with a smaller radius than the average penumbra. I created a mask of all points inside the penumbra above a threshold of 1.2 times the continuum intensity outside the spot 


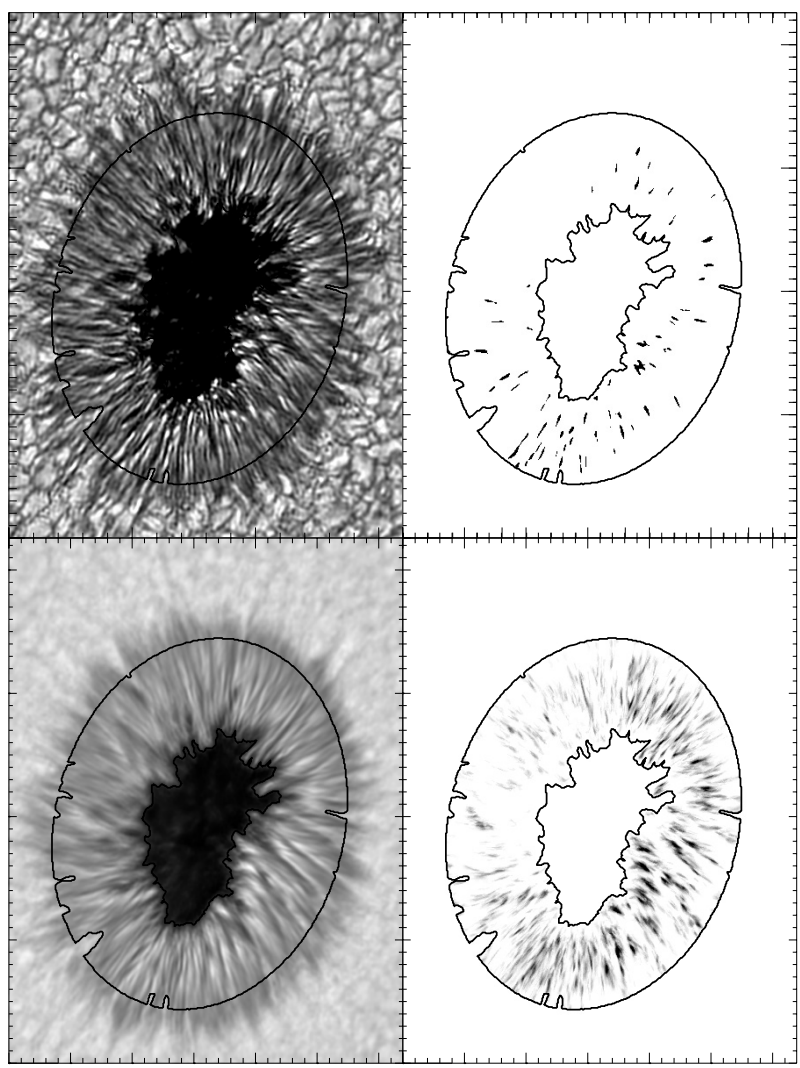

Fig. C.1. Overview of the DOT data set. Top row: single intensity image (left) with corresponding mask of brightenings (right). Bottom row: temporal averages over the time series. The black contours outline the area considered to be the penumbra. Tick marks are in arcsec.

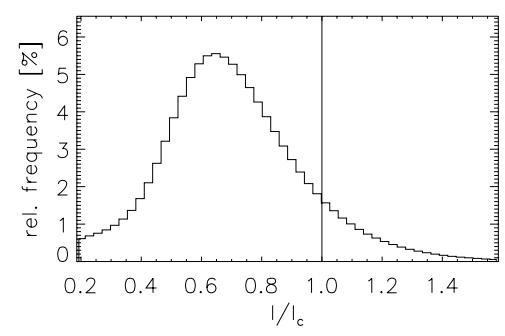

Fig. C.2. Average histogram of penumbral intensities in the time series.

for each of the 214 images of the time series. The number and area of the PGs were taken from the statistics of the individual masks.

On a single radial cut starting at the center and ending at the outer penumbral boundary, usually two or three individual PGs can be detected, even if they do not always all exceed the threshold used. The temporal average of the masks clearly shows the dependence of the PGs on the azimuthal position inside the spot: on the limb side, the PGs appear roundish and have a much higher frequency than for azimuths of $90^{\circ}$, respectively, $270^{\circ}$, where they usually appear as thin streaks. On the center side, they are almost completely missing. As in a single image, usually two or more PGs can be seen for any radial cut in the temporal average. The intensity of all points inside the boundaries defined above during the time series yielded the histogram of Fig. C.2, which shows that around $10 \%$ of all points are on average brighter than the outside continuum intensity.

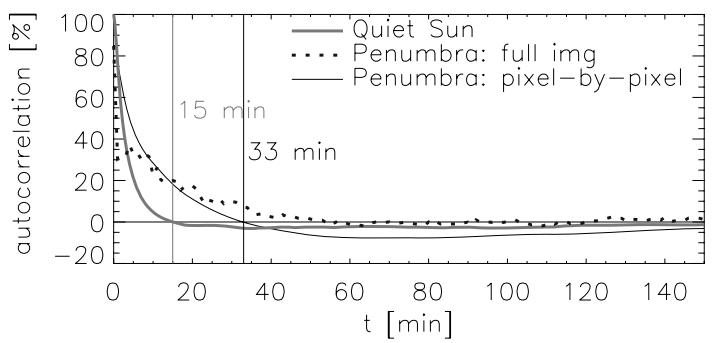

Fig. C.3. Autocorrelation functions of the intensity in the penumbra and a quiet Sun reference area.

All subsequent statistics and quantities were derived from the full time series in the area defined as belonging to the penumbra. On average, in each image of the time series I found around 90 penumbral grains with an intensity above 1.2 times the continuum intensity outside the spot. The average size of the PGs was 16 pixels, which would correspond to a square area of $200 \times 200 \mathrm{~km}^{2}$. They covered an area fraction of around $3 \%$ of the whole penumbra. Points with an intensity above a continuum intensity of unity covered $10 \%$ of the full area.

The intensity patterns in the penumbra disappear after a lifetime of around $30 \mathrm{~min}$; the autocorrelation drops to zero around that time (cf. Fig. C.3). For comparison, I used a granulation area outside the spot, which shows a characteristic correlation time of below $15 \mathrm{~min}$. I used two types of autocorrelation with the same result: autocorrelation of the full images (restricted to the penumbra), and pixel-by-pixel autocorrelation, where only the intensity of one spatial position with time was used in the derivation of the autocorrelation. The number of PGs is, however, constant to a high degree. Thus, I conclude that the rate of the appearance of PGs should also have a characteristic timescale of around $30 \mathrm{~min}$. 